\title{
X-ray spectroscopy of NGC 5548
}

\author{
J. S. Kaastra ${ }^{1}$, K. C. Steenbrugge ${ }^{1}$, A. J. J. Raassen ${ }^{1,2}$, R. L. J. van der Meer ${ }^{1}$, A. C. Brinkman ${ }^{1}$, \\ D. A. Liedahl ${ }^{3}$, E. Behar ${ }^{4}$, and A. de Rosa ${ }^{5}$ \\ 1 SRON National Institute for Space Research Sorbonnelaan 2, 3584 CA Utrecht, The Netherlands \\ 2 Astronomical Institute "Anton Pannekoek", Kruislaan 403, 1098 SJ Amsterdam, The Netherlands \\ 3 Physics Department, Lawrence Livermore National Laboratory, PO Box 808, L-11, Livermore, CA 94550, USA \\ 4 Department of Physics, Columbia University, 550 West 120th Street, New York, NY 10027, USA \\ 5 Istituto di Astrofisica Spaziale, CNR, Via del Fosso del Cavaliere 100, Roma 00133, Italy
}

Received 14 December 2001 / Accepted 12 February 2002

\begin{abstract}
We analyze the high-resolution X-ray spectrum of the Seyfert 1 galaxy NGC 5548, for the full 0.1-10 keV band, using improved calibration results of the Chandra-LETGS instrument. The warm absorber consists of at least three ionization components, with a low, medium and high ionization parameter. The X-ray absorbing material, from an outflowing wind, covers the full range of velocity components found from UV absorption lines. The presence of redshifted emission components for the strongest blue-shifted resonance absorption lines indicate that the absorber is located at a distance larger than the edge of the accretion disk. We derive an upper limit to the edge of the accretion disk of 1 light year. Absorption lines from ions of at least ten chemical elements have been detected, and in general there are no strong deviations from solar abundances. The narrow emission lines from the $\mathrm{O}$ VII and NeIX forbidden and intercombination lines probably originate from much larger distances to the black hole. We find evidence for weak relativistically broadened oxygen and nitrogen emission lines from the inner parts of the accretion disk, but at a much smaller flux level than those observed in some other active galactic nuclei. In addition, there is a broad, non-relativistic C VI Ly $\alpha$ emission line that is consistent with emission lines from the inner part of the optical/UV broad line region.
\end{abstract}

Key words. galaxies: individual: NGC 5548 - galaxies: Seyfert - quasars: absorption lines quasars: emission lines - X-rays: galaxies

\section{Introduction}

Active Galactic Nuclei (AGN) show a rather violent environment. Gas is being swallowed by the black hole, which is fed by a continuous supply of fresh material through the accretion disk. This process becomes visible as intense high-energy radiation from the inner parts of the disk and the immediate surroundings of the black hole. This radiation field may drive outflows from the nucleus. The detection of these outflows allows us to probe into the inner nuclear regions.

At least $50 \%$ of Seyfert 1 galaxies exhibit signatures of photo-ionized gas in their X-ray spectra (the so-called "warm absorber", e.g. Reynolds 1997; George et al. 1998). In previous X-ray (e.g., ASCA) spectra the most prominent features were identified as O VII and O VIII absorption edges. However, these spectra did not have the

Send offprint requests to: J. S. Kaastra,

e-mail: J.Kaastra@sron.nl energy resolution to determine velocity shifts smaller than $\sim 3000 \mathrm{~km} \mathrm{~s}^{-1}$, or to perform any line diagnostics. Until recently, the only information regarding the dynamics of circum-nuclear material along the line of sight has been from the detection of UV absorption features. These invariably indicate outflows with velocities of hundreds of $\mathrm{km} \mathrm{s}^{-1}$ (e.g. Crenshaw et al. 1999). Despite having a high geometrical covering factor, the origin, location, velocity field, and ionization structure of the X-ray absorber/emitter remained poorly understood for nearly two decades. In particular the relationship between the X-ray and UV absorbers has presented considerable problems for formulating a consistent picture of the inner nucleus in active galaxies. Only recently sufficiently high spectral resolution for the study of the inner nucleus has become available in the X-ray band thanks to the Chandra and XMM-Newton grating spectrometers. The first Chandra spectrum of a Seyfert 1 galaxy (NGC 5548, Kaastra et al. 2000) showed a wealth of discrete absorption lines. 


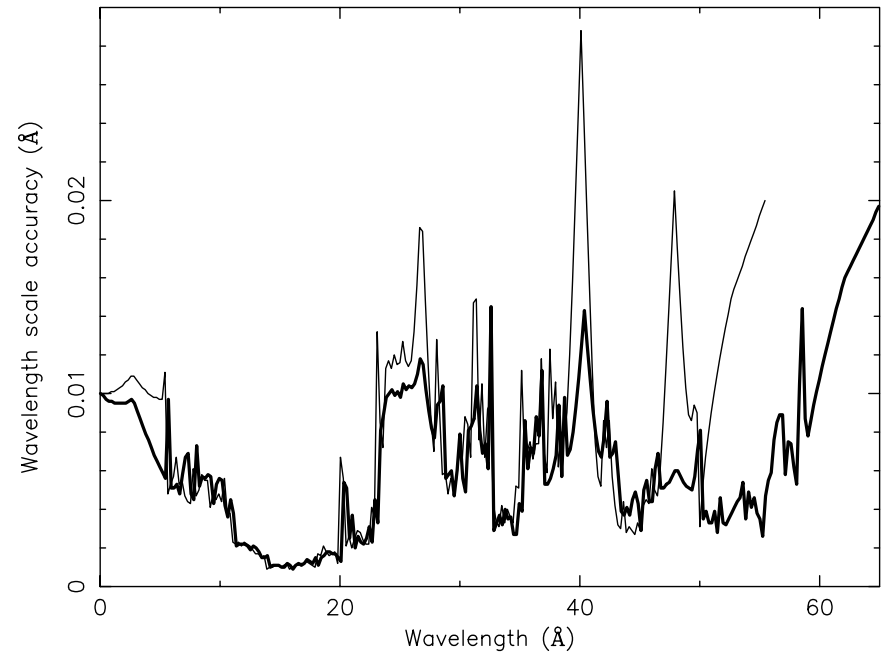

Fig. 1. Accuracy of the wavelength scale for the central plate of the HRC-S detector, for -1 order (thin line) and +1 order (thick line).

Here we reanalyze the Chandra data of NGC 5548 using significantly improved wavelength and effective area calibration for the LETGS instrument. We also extend the analysis to the full wavelength range and analyze the data with a global spectral fitting method.

The layout of the paper is as follows. We first discuss the data analysis in Sect. 2. In Sect. 3 we characterize the global continuum spectrum, and in Sect. 4 we analyze individual absorption and emission line features. In Sect. 5 we present the results of our global fit as well as the subsequent analysis of column densities in terms of photoionization models. We discuss our results in Sect. 6 .

\section{Data analysis}

\subsection{LETGS data}

The present Chandra observations of NGC 5548 were obtained on December 11/12, 1999, with an effective exposure time of $86400 \mathrm{~s}$. We used the High Resolution Camera (HRC-S) in combination with the Low Energy Transmission Grating (LETG). The spectral resolution of the instrument is about $40 \mathrm{~m} \AA$ and almost constant over the entire wavelength range (1.5-180 ̊). Event selection and background subtraction were done using the same standard processing as used for the first-light observation of Capella (Brinkman et al. 2000).

In our first analysis (Kaastra et al. 2000) the wavelength scale was accurate to within $15 \mathrm{~m} \AA$. We were able to improve this accuracy by an order of magnitude in several important regions of the spectrum by using the strongest spectral lines of a long Capella observation. Due to the dithering of the instrument, these monochromatic lines sweep over the detector surface, thereby allowing us to map the detector non-linearities. Full details will be described elsewhere (Van der Meer et al. 2002). In Fig. 1 we show the estimated accuracy of our wavelength scale. Fortunately, the strong spectral line features of NGC 5548 correspond to wavelengths for which Capella has strong emission lines. This allows for an accurate wavelength scale in the region with spectral features.

The line spread function of the instrument is well approximated by the square of a Lorentzian, with a wavelength dependent width. This shape and the relevant parameters were derived from the ground calibration and verified in-flight using Capella data.

In our first analysis the effective area calibration of the LETGS had not yet been finished. Our efficiency estimates were based upon pre-flight estimates for wavelengths below $60 \AA$ and on in-flight calibration using data from Sirius B for the longer wavelengths. Since then a better calibration has been obtained using LETGS observations of the white dwarf HZ 43 and simultaneous XMM/Chandra observations of the BL Lac object PKS 2155-304. The absolute effective area is accurate to about $10 \%$. The relative effective area (on the $\AA$ scale) is accurate to within a few percent, without significant small scale variations.

The relative strength of the higher spectral orders were derived from a combination of ground calibration and inflight calibration of Capella. In our case the combined continuum produced by the higher orders becomes comparable to the first order continuum at wavelengths above $83 \AA$. At these wavelengths our signal is already very weak due to the Galactic absorption and high background. At $83 \AA$ the subtracted background is ten times the first order signal, but since the background as a function of wavelength is rather smooth, its main effect is merely an increased noise level rather than a source of systematic uncertainty. Below $64 \AA$ the subtracted background is smaller than the measured continuum.

More details about the calibration are given in Van der Meer et al. (2002).

A response matrix was generated including higher spectral orders up to the \pm 10 th order for the further analysis. All subsequent spectral fitting was done using the SPEX package (Kaastra et al. 1996). All errors quoted in this paper are $1 \sigma$ errors.

\subsection{HETGS data}

NGC 5548 was observed with the HETGS instrument of Chandra two months after our LETGS observation (Yaqoob et al. 2001; George 2001). We have extracted the spectrum from the Chandra archive and processed it using the standard CIAO pipeline. The exposure time of this HETGS observation was similar to the exposure time of the LETGS. However the source was two times fainter during the HETGS observation. This combined with the smaller effective area of the HETGS at longer wavelengths makes a comparison with our LETGS observations difficult. We have folded our best-fit warm absorber model (see Sect. 4) through the HETGS response matrix and allowed only the underlying continuum parameters to vary (power law and modified blackbody). We find no evidence 


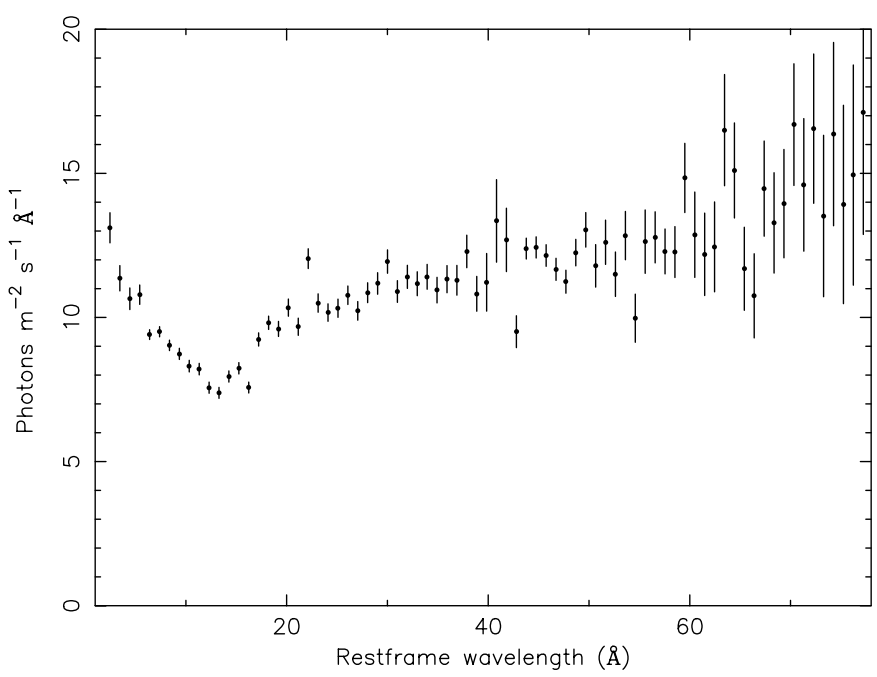

Fig. 2. Fluxed, higher order and background subtracted LETGS spectrum of NGC 5548, binned to $1 \AA$ bins and corrected for cosmological redshift and Galactic absorption.

for large differences between our model and the HETGS data. Therefore we do not discuss the HETGS data in full detail. We only use it where it can supplement the LETGS data, in particular for the profiles of the short wavelength lines and some emission features.

\subsection{BeppoSAX data}

BeppoSAX observed NGC 5548 simultaneously with the LETGS. The BeppoSAX data are especially useful for monitoring the continuum variability, the Fe-K complex and the high energy cut-off. Since the main aim of our paper is high resolution X-ray spectroscopy we do not discuss these BeppoSAX data in the present paper.

\section{Continuum spectrum}

\subsection{Fluxed spectrum}

As a first step we present in Fig. 2 a fluxed spectrum of NGC 5548. Higher spectral orders have been subtracted using a bootstrap method and the spectrum has been corrected for cosmological redshift $(z=0.01676$, Crenshaw et al. 1999) and Galactic absorption (column density $\left.1.65 \times 10^{24} \mathrm{~m}^{-2}\right)$.

\subsection{Fitting the continuum}

The continuum spectrum cannot be fit satisfactorily with a simple power law model. The broad dip in the spectrum around $15 \AA$ clearly contradicts such a picture. This dip has been attributed in the past to continuum absorption edges mainly from O VII and O VIII. However, the analysis of the absorption lines as presented by Kaastra et al. (2000) and refined in the present work shows that the continuum edges are much weaker. The dip must be attributed therefore to the high-energy cut-off of the soft

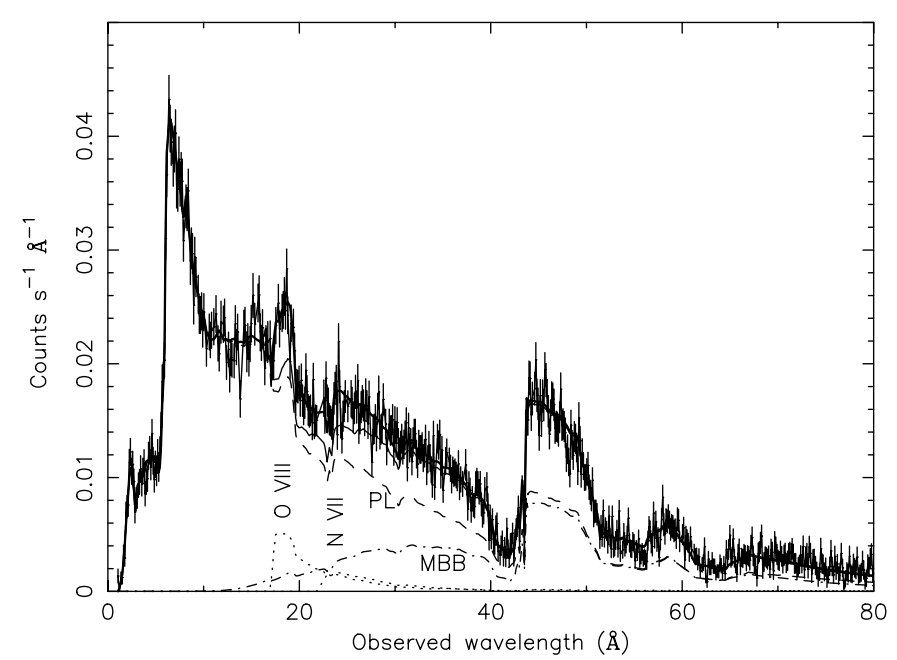

Fig. 3. Chandra LETGS spectrum of NGC 5548, with absorption lines excluded and in this plot binned by a factor of 4 in order to have a better display of the continuum shape. The contribution of the power law (PL) and modified blackbody (MBB) components are indicated by the dashed and dashdotted lines. The sum of these two components is the thin solid line. The contribution from the broadened O VIII and N VII lines (dotted lines) are indicated separately, and the total continuum is indicated by the thicker solid line.

excess component. We therefore add a modified blackbody component (MBB) to the spectrum, in addition to the power law component, similar to the model used by Kaastra \& Barr (1989) in their analysis of the EXOSAT data of NGC 5548 .

For a proper analysis of the continuum spectrum it is important to account for the strongest absorption lines. Therefore in our initial fit we excluded the spectral regions where the absorption lines are. If these lines are not taken into account in the modeling (i.e., not excluded), the normalization of the continuum is $5-10 \%$ too small, and the power law photon index is 0.05 smaller. The lines that were excluded are given in Table 2 . The actual fit was done using the full resolution count spectrum.

The best fit to this continuum spectrum (including weak broad emission line features as discussed below) has a $\chi^{2}$ of 1986 for 1834 degrees of freedom (Table 1). The spectrum is shown in Fig. 3. For the temperature of the modified blackbody component we obtained a value of $0.095 \pm 0.004 \mathrm{keV}$, the same value as derived for the EXOSAT data of July 1984 as presented by Kaastra \& Barr (1989). Also the power law normalization and photon index are the same as in July 1984. However the derived normalization of the modified blackbody component is half the value measured by EXOSAT.

NGC 5548 was slightly variable during the LETGS observation. The source showed a smooth $\sim 50 \%$ increase from start to end (see Fig. 4). From an analysis of the hardness ratio in the LETGS data we find that the spectrum softens when the source becomes brighter. The photon index increases from typically 1.65 at the start of the observation to 1.80 at the end. The same tendency is 
Table 1. Fits to the continuum only, excluding lines.

\begin{tabular}{lr}
\hline \hline Parameter & value \\
\hline$\chi^{2}$ & 1986 \\
degrees of freedom & 1834 \\
Power law photon index $\Gamma$ & $1.731 \pm 0.028$ \\
Power law normalization $^{\mathrm{a}}$ & $106.0 \pm 2.0$ \\
$2-10$ keV Flux $\left(\mathrm{W} \mathrm{m}^{-2}\right)$ & $4.0 \times 10^{-14}$ \\
MBB Temperature $(\mathrm{keV})$ & $0.095 \pm 0.004$ \\
MBB Normalization & $(2.3 \pm 0.4) \times 10^{33}$ \\
$\mathrm{C}$ VI Ly $\alpha^{\mathrm{c}} 33.736 \AA$ & $0(<3)$ \\
$\mathrm{N}$ VII Ly $\alpha^{\mathrm{c}} 24.781 \AA$ & $12 \pm 5$ \\
O VIII Ly $\alpha^{\mathrm{c}} 18.969 \AA$ & $19(-6,+10)$ \\
$\mathrm{C}$ VI Ly $\alpha^{\mathrm{d}} 33.736 \AA$ & $<0.3$ \\
$\mathrm{~N}$ VII Ly $\alpha^{\mathrm{d}} 24.781 \AA$ & 1.3 \\
O VIII Ly $\alpha^{\mathrm{d}} 18.969 \AA$ & 2.3 \\
Inclination $i($ degrees $)$ & $46(-3,+8)$ \\
Inner radius $r_{1}^{\mathrm{e}}$ & $40(-0.4,+1.0)$ \\
Outer radius $r_{2}^{\mathrm{e}}$ & $0.0(-0.0,+1.2)$ \\
Scale height $h^{\mathrm{d}}$ & $3.9 \pm 0.6$ \\
Emissivity index $q^{-1}$ &
\end{tabular}

a In photons $\mathrm{m}^{-2} \mathrm{~s}^{-1} \mathrm{keV}^{-1}$ at $1 \mathrm{keV}$.

b Emitting area $A$ times square root of electron density $n_{\mathrm{e}}$ in $\mathrm{m}^{0.5}$.

c Observed line intensities in units of photons $\mathrm{m}^{-2} \mathrm{~s}^{-1}$ corrected for Galactic absorption.

d Equivalent width in $\AA$.

e In units of $G M / c^{2}$.

confirmed by simultaneous BeppoSAX observations. These BeppoSAX observations show that the source was at a minimum when the LETGS observation started. Since the changes in the shape of the continuum are not very large, even though the flux increased by $\sim 50 \%$, we do not investigate the time variability any further here. The statistical quality of the spectrum does not allow us to check for time variability of narrow spectral features.

Finally, we note that during the HETGS observation of NGC 5548 the source showed no significant variability at all.

\subsection{Broad emission lines}

We also tested for the possible presence of relativistically broadened Lyman alpha lines from oxygen, nitrogen and carbon. Such lines were recently discovered in the XMM-Newton spectra of two narrow line Seyfert 1 galaxies (Branduardi-Raymont et al. 2001). We added three narrow emission lines at the rest wavelengths of the $\mathrm{C}$ VI, $\mathrm{N}$ viI and O viII Ly $\alpha$ lines to the model. These lines were then convolved with the relativistic disk line profile of Laor (1991). Parameters of this disk model are the disk inner and outer radii $r_{1}$ and $r_{2}$, its inclination $i$ and the emissivity law that we take is proportional to $\left(r^{2}+h^{2}\right)^{-q / 2}$ with $q$ the emissivity power index at large radii $r$ and $h$ a scale height.

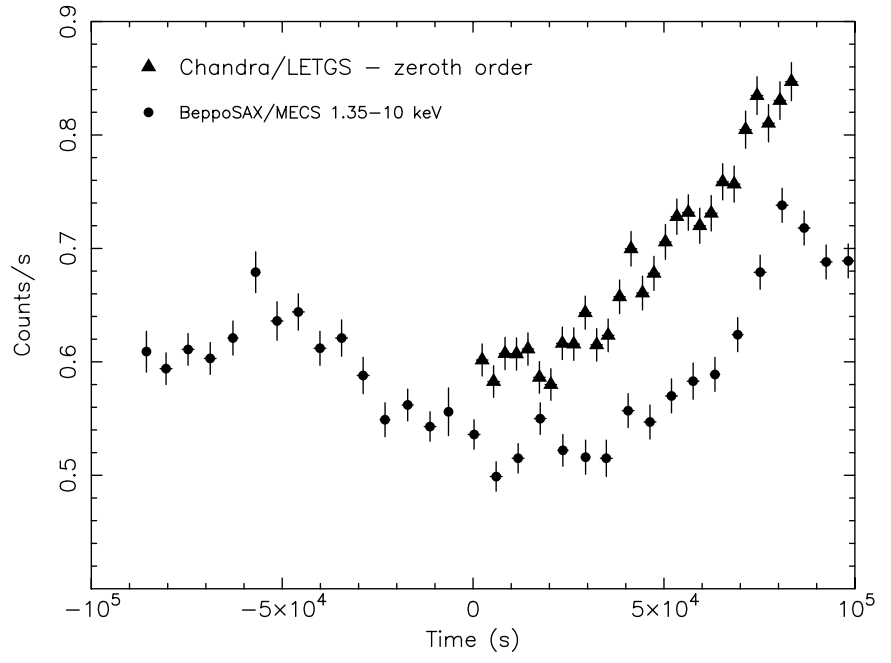

Fig. 4. Light curve of NGC 5548 for the zeroth spectral order of the LETGS and the BeppoSAX MECS data. Bin size of the LETGS data is $3000 \mathrm{~s}$, the MECS data have been averaged per satellite orbit. Zero point is the start time of the LETGS data.

Our fit improved ( $\chi^{2}$ decreases from 2083 to 1986 with 8 degrees of freedom less) by including the oxygen and nitrogen lines. We found no evidence for significant carbon emission with the same profile (but see below). The best fit parameters for the continuum and broad lines are shown in Table 1.

Inspecting the fit residuals of a global fit to the spectrum including the narrow absorption lines (Sect. 5.2) we find evidence for excess emission around the CVI Ly $\alpha$ line at $33.736 \AA$ (see Fig. 13). This broad feature can be approximated in the rest frame of NGC 5548 with a Gaussian profile centered at $33.84 \pm 0.26 \AA$, a width $(\sigma)$ of $0.51 \pm 0.16 \AA$, corresponding to $\sigma_{\mathrm{v}}=4500 \pm$ $1400 \mathrm{~km} \mathrm{~s}^{-1}$, and a flux of $5.6_{-1.7}^{+3.5}$ photons $\mathrm{m}^{-2} \mathrm{~s}^{-1}$, corrected for Galactic absorption. The width is significantly smaller than the width of a relativistic line based upon the best fit model for the O VIII and N viI Ly $\alpha$ lines.

\section{Narrow absorption lines}

\subsection{Line identifications}

As a next step we study the narrow absorption lines. We determined the line centroids and equivalent widths of the most prominent features actually seen in the spectrum as well as for some important lines that are expected to be present but are weak. Therefore some lines have a poorly determined equivalent width. These lines are listed with their Doppler velocity (Sect. 4.2) and equivalent width in Table 2.

We included the $\mathrm{O}$ vi KLL $1 \mathrm{~s} 2 \mathrm{~s} 2 \mathrm{p}$ resonances at 21.87 and $22.05 \AA$ that were recently calculated by Pradhan (2000). The slight redshift of the OVIII Ly $\gamma$ line at $15.176 \AA$ is possibly due to contamination by FexVII at $15.265 \AA$.

There is a problem with the Ne IX $1 \mathrm{~s}^{2}-1 \mathrm{~s} n \mathrm{p}$ resonance lines. The $n=2$ line at $13.447 \AA$ is clearly detected. 
Table 2. Absorption lines in NGC 5548. Lines significantly blended by lines from other ions are indicated by a *; the equivalent widths in this case are not corrected for blending. Unresolved line blends of the same ion are indicated by "blend".

\begin{tabular}{|c|c|c|}
\hline $\begin{array}{l}\text { Rest frame } \\
\text { Wavelength } \\
(\AA) \\
\end{array}$ & $\begin{array}{r}\text { velocity } \\
\left.100 \mathrm{~km} \mathrm{~s}^{-1}\right) \\
\end{array}$ & $\begin{array}{l}\text { equiv. identification } \\
\text { width } \\
(\mathrm{m} \AA) \\
\end{array}$ \\
\hline $\begin{array}{l}40.268 \\
34.973\end{array}$ & $\begin{array}{l}-3.7 \pm 1.6 \\
-2.9 \pm 3.3\end{array}$ & $\begin{array}{r}136 \pm 41 \mathrm{C} \mathrm{V} 1 \mathrm{~s}^{2}-1 \mathrm{~s} 2 \mathrm{p}{ }^{1} \mathrm{P}_{1} \\
26 \pm 22 \mathrm{C} \mathrm{V} 1 \mathrm{~s}^{2}-1 \mathrm{~s} 3 \mathrm{p}{ }^{1} \mathrm{P}_{1}\end{array}$ \\
\hline $\begin{array}{l}33.736 \\
28.466 \\
26.990\end{array}$ & $\begin{array}{l}-4.3 \pm 1.2 \\
-5.1 \pm 1.3 \\
-3.1 \pm 2.7\end{array}$ & $\begin{array}{l}90 \pm 30 \text { C VI } 1 \mathrm{~s}-2 \mathrm{p}(\mathrm{Ly} \alpha) \\
28 \pm 11 \text { C VI } 1 \mathrm{~s}-3 \mathrm{p}(\mathrm{Ly} \beta) \\
15 \pm 11 \text { C VI } 1 \mathrm{~s}-4 \mathrm{p}(\mathrm{Ly} \gamma)\end{array}$ \\
\hline $\begin{array}{l}28.787 \\
24.898 \\
\end{array}$ & $-3.3 \pm 3.6$ & $\begin{array}{r}44 \pm 24 \text { N VI } 1 \mathrm{~s}^{2}-1 \mathrm{~s} 2 \mathrm{p}{ }^{1} \mathrm{P}_{1} \\
8 \pm 12 \text { N VI } 1 \mathrm{~s}^{2}-1 \mathrm{~s} 3 \mathrm{p}{ }^{1} \mathrm{P}_{1} \\
\end{array}$ \\
\hline $\begin{array}{l}24.781 \\
20.910\end{array}$ & $-2.9 \pm 1.9$ & $\begin{array}{r}66 \pm 12 \text { N VII } 1 \mathrm{~s}-2 \mathrm{p}(\operatorname{Ly} \alpha) \\
4 \pm 11 \text { N VII } 1 \mathrm{~s}-2 \mathrm{p}(\operatorname{Ly} \beta)\end{array}$ \\
\hline $\begin{array}{l}22.05 \\
21.87\end{array}$ & $-7.1 \pm 2.0$ & $\begin{array}{l}17 \pm 11 \text { O VI } 1 \mathrm{~s}^{2} 2 \mathrm{~s}-1 \mathrm{~s} 2 \mathrm{p}\left({ }^{1} \mathrm{P}\right) 2 \mathrm{~s} \\
10 \pm 12 \text { O VI } 1 \mathrm{~s}^{2} 2 \mathrm{~s}-1 \mathrm{~s} 2 \mathrm{p}\left({ }^{3} \mathrm{P}\right) 2 \mathrm{~s}\end{array}$ \\
\hline $\begin{array}{l}21.602 \\
18.627 \\
17.768 \\
17.396 \\
17.200\end{array}$ & $\begin{array}{l}-7.8 \pm 2.1 \\
-4.4 \pm 1.0 \\
-5.6 \pm 2.7 \\
-3.5 \pm 3.5\end{array}$ & $\begin{array}{r}60 \pm 12 \text { O VII } 1 \mathrm{~s}^{2}-1 \mathrm{~s} 2 \mathrm{p}{ }^{1} \mathrm{P}_{1} \\
34 \pm 6 \text { O VII } 1 \mathrm{~s}^{2}-1 \mathrm{~s} 3 \mathrm{p}{ }^{1} \mathrm{P}_{1} \\
19 \pm 8 \text { O VII } 1 \mathrm{~s}^{2}-1 \mathrm{~s} 4 \mathrm{p}{ }^{1} \mathrm{P}_{1} \\
21 \pm 9 \text { O VII } 1 \mathrm{~s}^{2}-1 \mathrm{~s} 5 \mathrm{p}{ }^{1} \mathrm{P}_{1} \\
12 \pm 9 \text { O VII } 1 \mathrm{~s}^{2}-1 \mathrm{~s} 6 \mathrm{p}{ }^{1} \mathrm{P}_{1}\end{array}$ \\
\hline $\begin{array}{l}18.969 \\
16.006 \\
15.176 \\
14.821\end{array}$ & $\begin{array}{l}-5.4 \pm 1.0 \\
-0.4 \pm 1.1 \\
+3.8 \pm 2.0\end{array}$ & 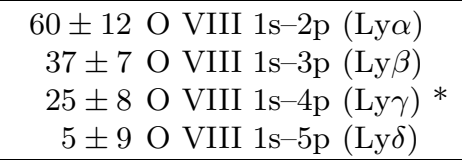 \\
\hline $\begin{array}{l}13.447 \\
11.544 \\
11.001 \\
10.765 \\
\end{array}$ & $\begin{array}{l}-2.2 \pm 1.6 \\
-6.0 \pm 2.7 \\
-2.0 \pm 3.3 \\
\end{array}$ & $\begin{array}{l}38 \pm 7 \text { Ne IX } 1 \mathrm{~s}^{2}-1 \mathrm{~s} 2 \mathrm{p}{ }^{1} \mathrm{P}_{1} \\
0 \pm 10 \text { Ne IX } 1 \mathrm{~s}^{2}-1 \mathrm{~s} 3 \mathrm{p}{ }^{1} \mathrm{P}_{1} * \\
21 \pm 8 \text { Ne IX } 1 \mathrm{~s}^{2}-1 \mathrm{~s} 4 \mathrm{p}{ }^{1} \mathrm{P}_{1} * \\
16 \pm 8 \text { Ne IX } 1 \mathrm{~s}^{2}-1 \mathrm{~s} 5 \mathrm{p}{ }^{1} \mathrm{P}_{1} *\end{array}$ \\
\hline $\begin{array}{l}12.134 \\
10.238\end{array}$ & $-8.1 \pm 1.7$ & $\begin{array}{l}38 \pm 7 \text { Ne X 1s-2p }(\operatorname{Ly} \alpha)^{*} \\
-4 \pm 10 \text { Ne X 1s-2p }(\operatorname{Ly} \beta)\end{array}$ \\
\hline $\begin{array}{l}11.003 \\
10.025 \\
\end{array}$ & $-6.5 \pm 2.7$ & $\begin{array}{l}21 \pm 8 \mathrm{Na} \text { X } 1 \mathrm{~s}^{2}-1 \mathrm{~s} 2 \mathrm{p}{ }^{1} \mathrm{P}_{1} * \\
22 \pm 8 \mathrm{Na} \text { XI } 1 \mathrm{~s}-2 \mathrm{p}(\mathrm{Ly} \alpha)\end{array}$ \\
\hline $\begin{array}{l}9.169 \\
8.421 \\
\end{array}$ & & $\begin{array}{l}12 \pm 8 \mathrm{Mg} \text { XI } 1 \mathrm{~s}^{2}-1 \mathrm{~s} 2 \mathrm{p}{ }^{1} \mathrm{P}_{1} \\
17 \pm 7 \mathrm{Mg} \text { XII } 1 \mathrm{~s}-2 \mathrm{p}(\mathrm{Ly} \alpha)\end{array}$ \\
\hline $\begin{array}{l}61.050 \\
43.763 \\
6.648 \\
6.182 \\
\end{array}$ & $\begin{array}{l}-4.1 \pm 2.4 \\
-3.0 \pm 1.9\end{array}$ & $\begin{array}{r}129 \pm 74 \text { Si VIII } 2 \mathrm{p}-3 \mathrm{~d} \\
35 \pm 17 \text { Si XI } 2 \mathrm{~s}^{2}-2 \mathrm{~s} 3 \mathrm{p} \\
12 \pm 6 \text { Si XIII } 1 \mathrm{~s}^{2}-1 \mathrm{~s} 2 \mathrm{p}{ }^{1} \mathrm{P}_{1} \\
12 \pm 6 \text { Si XIV } 1 \mathrm{~s}-2 \mathrm{p}(\mathrm{Ly} \alpha)\end{array}$ \\
\hline $\begin{array}{l}42.517 \\
36.398\end{array}$ & $\begin{array}{l}-8.5 \pm 2.3 \\
-3.5 \pm 3.2\end{array}$ & $\begin{array}{r}161 \pm 50 \text { S X } 2 \mathrm{p}-3 \mathrm{~d} \\
37 \pm 24 \text { S XII } 2 \mathrm{p}-3 \mathrm{~d}\end{array}$ \\
\hline 58.963 & & $49 \pm 54$ Fe XIV 3p-4d \\
\hline 52.911 & $-8.5 \pm 1.7$ & $102 \pm 54$ Fe XV $3 s^{2}-3 s 4 p$ \\
\hline 38.956 & $-8.9 \pm 3.8$ & $37 \pm 26$ Fe XV $3 s^{2}-3 s 5 p$ \\
\hline 50.350 & $+1.7 \pm 2.4$ & $98 \pm 36$ Fe XVI $3 \mathrm{~s}-4 \mathrm{p} *$ \\
\hline 36.749 & $-6.2 \pm 2.5$ & $33 \pm 24$ Fe XVI $3 \mathrm{~s}-5 \mathrm{p}$ \\
\hline 15.014 & $-3.8 \pm 1.8$ & $23 \pm 8$ Fe XVII $2 p-3 d$ \\
\hline 12.123 & $-5.4 \pm 1.7$ & $26 \pm 9$ Fe XVII $2 \mathrm{p}-4 \mathrm{~d} *$ \\
\hline 11.251 & & $10 \pm 10$ Fe XVII $2 p-5 d$ \\
\hline 10.771 & $-3.6 \pm 3.3$ & $16 \pm 8$ Fe XVII $2 \mathrm{p}-6 \mathrm{~d}$ \\
\hline 13.826 & $-5.0 \pm 2.2$ & $22 \pm 8$ Fe XVII $2 p-3 p$ \\
\hline 14.207 & $-5.7 \pm 2.7$ & $15 \pm 8$ Fe XVIII $2 \mathrm{p}-3 \mathrm{~d}$ blend \\
\hline 13.522 & $-3.1 \pm 2.4$ & $27 \pm 8$ Fe XIX $2 p-3 d$ blend \\
\hline 12.837 & & $35 \pm 16$ Fe XX $2 p-3 d$ blend \\
\hline 12.286 & $+2.0 \pm 3.4$ & $17 \pm 8$ Fe XXI $2 p-3 d *$ \\
\hline 11.706 & $+2.8 \pm 3.6$ & $13 \pm 9$ Fe XXII $2 p-3 d$ \\
\hline
\end{tabular}

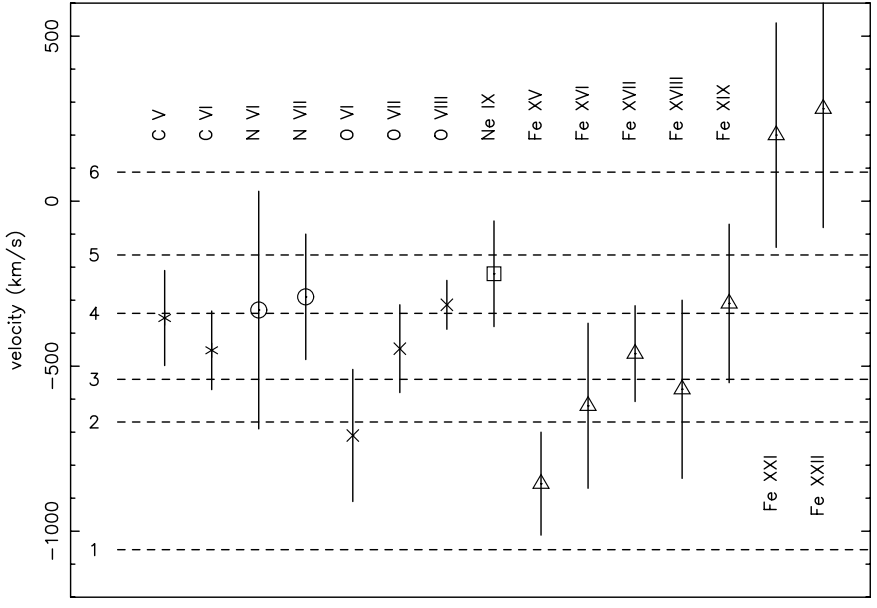

Fig. 5. Average velocity with respect to the AGN rest frame for the X-ray absorption lines of different ions. The components $1-6$ indicate the velocity components identified in the UV by Crenshaw \& Kraemer (1999).

This line may have some contamination from the Fe XIX line at $13.453 \AA$. However, there is no significant detection of the $n=3$ line at $11.544 \AA$ (equivalent width $0 \pm 10 \mathrm{~m} \AA$ ). The line observed at $10.979 \AA$ can be either Nax $1 \mathrm{~s}^{2}-1 \mathrm{~s} 2 \mathrm{p}$ at $11.003 \AA$ or Ne IX $1 \mathrm{~s}^{2}-1 \mathrm{~s} 4 \mathrm{p}$ at $11.001 \AA$. However given the non-detection of the NeIX $n=3$ line we prefer the Na x identification. Similarly, we prefer to identify the line at $10.758 \AA$ with the FexVII line at $10.771 \AA$ instead of the Ne IX $1 \mathrm{~s}^{2}-1 \mathrm{~s} 5 \mathrm{p}$ line at $10.765 \AA$.

\subsection{Line shifts}

The improved wavelength calibration allows us to obtain more accurate outflow velocities. The systematic wavelength uncertainty in general is smaller than $0.01 \AA$, but several lines have systematic uncertainties of only 0.001-0.002 $\AA$ (see Fig. 1). Only in a few cases the systematic uncertainty reaches $0.02 \AA$, in particular near $60 \AA$ where Capella has no or only weak lines. For the majority of the lines, the statistical uncertainty of the line centroid dominates the systematic wavelength uncertainty. For consistency we have included the systematic errors in the entries of Table 2 .

We express the wavelength shifts as Doppler velocities with respect to the (optical narrow line region) redshift of 0.01676 . A negative sign indicates blue shift (outflow). For the weaker lines the Doppler velocity is not given since it is poorly constrained.

In Fig. 5 we plot the average velocity for the observed ions, including for each ion only those lines that are not suspected to be blended with other lines. For O viI we excluded the $1 s^{2}-1 s 2 p$ resonance line, since the average wavelength of this absorption line may be contaminated by its redshifted emission component. We also plot the velocities of the 6 velocity components identified in UV absorption lines by Crenshaw \& Kraemer (1999). 


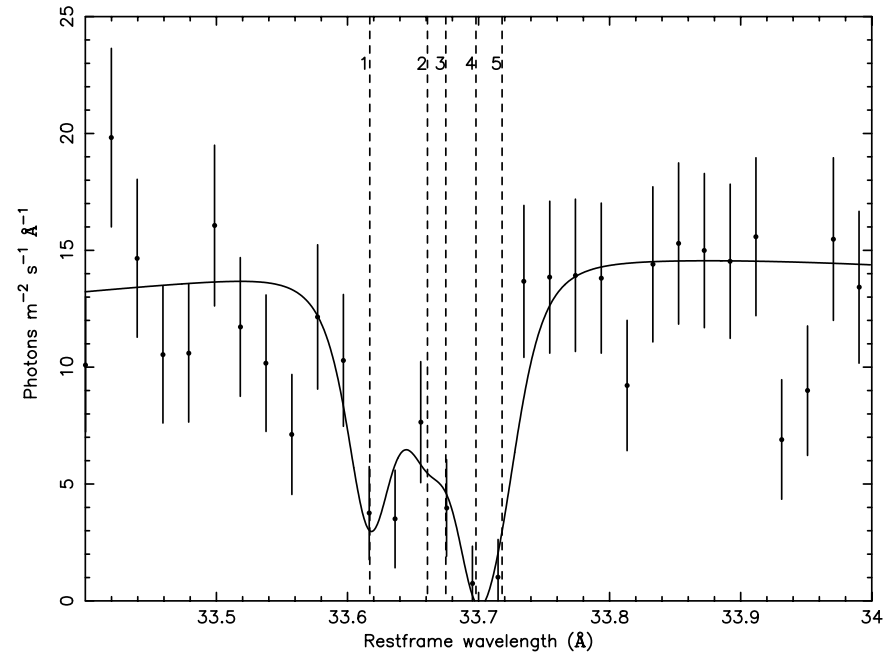

Fig. 6. The C vi Ly $\alpha$ line fitted using a sum of narrow line components with the velocities fixed at the values for the velocity components 1-5 identified in the UV by Crenshaw \& Kraemer (1999).

The component velocities are: $-1056,-669,-540,-340$, -163 and $+88 \mathrm{~km} \mathrm{~s}^{-1}$.

Most of the ions observed in the X-rays have velocities consistent with the velocity of component 4 $\left(-340 \mathrm{kms}^{-1}\right)$. However a velocity distribution between components $2-5$ is also possible. For the iron ions we see a clear tendency for less ionized ions to have larger outflow velocities than the more highly ionized ions. The same tendency is visible for the oxygen ions. A similar velocity pattern was also found in the XMM-Newton spectrum of the quasar IRAS $13349+2438$ by Sako et al. (2001).

\subsection{Line profiles and broadening}

As was shown by Kaastra et al. (2000) the measured Doppler width of the absorption lines was larger than the width derived from the intensity ratio of different lines from the same ion. This provides evidence that the lines are actually blends composed of different velocity components, with varying relative strength for each ion or group of ions. Our LETGS data have insufficient spectral resolution to resolve the blends fully, although we see evidence for broadening, differences in the centroids of the blends etc.

This is illustrated in Fig. 6, where we fitted the C VI Ly $\alpha$ line using a sum of narrow line components with the velocities fixed at the values for the velocity components 1-5 identified in the UV by Crenshaw \& Kraemer (1999). The C VI Ly $\alpha$ line is the best case to study here, since it is strong, lies in a region with good statistics and relatively high spectral resolution of the LETGS. It is also well isolated (not blended). In Table 3 we show the bestfit equivalent widths for the individual components. The centroids of these 5 components span only two times the instrumental $F W H M$. As a result there is a strong correla-
Table 3. C vi Ly $\alpha$ line.

\begin{tabular}{lrr}
\hline \hline Component & $\begin{array}{r}\text { velocity } \\
\mathrm{km} \mathrm{s}^{-1}\end{array}$ & $\begin{array}{r}\text { equivalent width } \\
\mathrm{m} \AA\end{array}$ \\
\hline 1 & -1056 & $37 \pm 10$ \\
2 & -669 & $19 \pm 26$ \\
3 & -540 & $0 \pm 31$ \\
4 & -340 & $37 \pm 21$ \\
5 & -163 & $20 \pm 15$ \\
Total & & $113 \pm 49$ \\
\hline
\end{tabular}

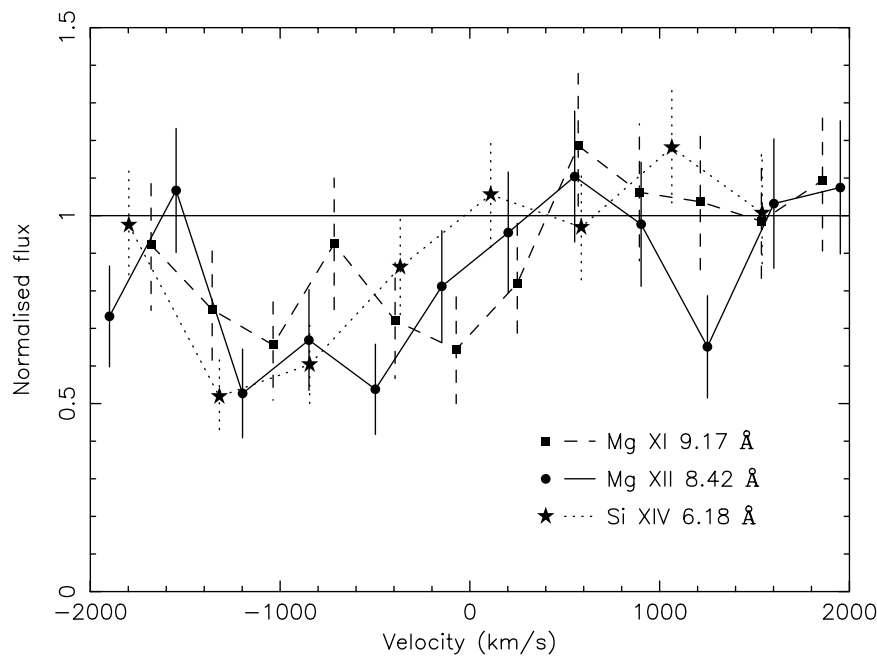

Fig. 7. Line profiles of the strongest lines in the MEG spectrum of NGC 5548. Fluxes have been normalized to 1 for the continuum. Bin size is $0.01 \AA$.

tions between these parameters and the equivalent widths are poorly constrained.

For comparison we also plot the velocity profiles of the strongest short wavelength lines as measured with the Medium Energy Grating (MEG) of the HETGS observation (Fig. 7). The equivalent widths of these absorption lines are within their error bars consistent with the values obtained from the LETGS in Table 2. The Mg lines have a significant contribution from the lower velocity gas components $2-5$ (few hundred $\mathrm{km} \mathrm{s}^{-1}$ outflow), but in addition the $\mathrm{Mg}$ lines and in particular the Si XIV Ly $\alpha$ line at $6.18 \AA$ have a strong component around $-1000 \mathrm{~km} \mathrm{~s}^{-1}$, consistent with component 1. Apparently Si XIV does not follow the general trend seen in the iron ions (Fig. 5).

Given this complex line structure, we may question if an analysis using a single line component in order to determine the column densities is sufficiently accurate.

In the UV, with 100 times better spectral resolution, the line blends are clearly resolved into 6 velocity components (Crenshaw \& Kraemer 1999) as we noted before. However, the individual components show further substructure. This is particularly evident from the profile of the dominant UV component (4) in C IV and N V. In their Fig. 1 this component has a rather "square", non-Gaussian appearance. Mathur et al. (1999) split this component into two parts. 
An important consideration is the number of velocity components taken into account, especially for saturated absorption lines. Consider for example a set of $n$ equal absorbers, each having a large column density $N$ that produces sufficient line opacity for the line core of each component to be saturated. If each absorber has the same velocity, the total equivalent width $W_{\text {tot }}$, due to the overlapping line profiles, is significantly smaller than $n$ times the equivalent width $W_{\text {ind }}$ for an individual component. But if the velocity separations of the components are larger than the intrinsic (turbulent or thermal) line width $\sigma_{\mathrm{v}}$, then $W_{\text {tot }}=n W_{\text {ind }}$.

In order to take this effect into account, we adopt the following procedure in the column density analysis of the next section. We split each line into 5 velocity components with equal column density for each component. These 5 components have the same intrinsic line broadening $\sigma_{\mathrm{v}}$ and have fixed velocities of $-163,-305,-375,-540$ and $-669 \mathrm{~km} \mathrm{~s}^{-1}$ corresponding to the UV components 5, 4a, 4b, 3 and 2 respectively, where we split the strongest and broadest component $4\left(-340 \mathrm{~km} \mathrm{~s}^{-1}\right)$ into two parts $4 \mathrm{a}$ and 4b, cf. Mathur et al. (1999). Of course this treatment ignores possible differences between the UV and X-ray lines, but since for most lines we do not have sufficient spectral resolution to resolve the profiles it is the best we can do with the present data set.

\section{Detailed spectral fitting}

In Sect. 4 we have identified the most prominent absorption lines. From the observed equivalent width ratio of these absorption lines it is possible to derive the column densities of the absorbing ions (e.g. Kaastra et al. 2000). There is good reason, however, to go one step further. First, the continuum opacity of the warm absorber appears to be small but not completely negligible. This may affect the estimate of the continuum spectrum. Further, some lines are significantly blended by lines from other ions resulting in uncertainties in the equivalent widths. Finally, some ions produce absorption lines that are too weak to be detected individually, but taken together these lines may represent a detectable signal. Moreover, column densities can also be constrained by lines that are predicted to be present but are not detected. Therefore we fitted the complete LETGS spectrum starting with the continuum model of Sect. 3 with the addition of a warm absorber model as described below.

\subsection{Slab model}

We generated a new model, named slab, for the SPEX package that treats in a simplified manner the absorption by a thin slab composed of different ions. The transmission $T(\lambda)$ of this slab is simply calculated as $T(\lambda)=$ $\exp \left[-\tau_{\mathrm{c}}(\lambda)-\tau_{\mathrm{l}}(\lambda)\right]$ with $\tau_{\mathrm{c}}$ and $\tau_{1}$ the total continuum and line optical depth, respectively. As long as the thickness of the slab is not too large, this most simple approxima- tion allows a fast computation of the spectrum, which is desirable for spectral fitting.

The continuum opacities are taken from Verner \& Yakovlev (1995). Line opacities and wavelengths for most ions are from Verner et al. (1996), with the following additions. For O VI we added the KLL resonances near $22 \AA$ as calculated by Pradhan (2000). For the L-shell ions of iron (Fe XVII-Fe XXIV) we used the HULLAC calculations provided by one of us (Liedahl). The wavelengths of these Lshell transitions were adjusted according to Phillips et al. (1999). Also the L-shell ions of Si vIII-Si XII, S X-S XIV and ArXv were calculated using the HULLAC code. In addition, we added the $2 \mathrm{p}-3 \mathrm{~d}$ inner shell resonance lines of Fe VII-Fe XV that we have calculated using the Cowan code (1981). These data were compared with those in Behar et al. (2001b) and found to be in reasonable agreement. These inner shell resonance lines occur mainly in the 16-17 $\AA$ band and appear to be extremely important diagnostic tools, since the normal resonance lines of these lowly ionized iron ions have their wavelengths mainly in the inaccessible EUV band. Their importance for active galactic nuclei was first demonstrated by Sako et al. (2001).

Each absorption line is then split into different velocity components, using

$\tau_{1}(v)=\sum_{i} \tau_{i} \exp \left[-\left(v-v_{i}\right)^{2} / 2 \sigma_{\mathrm{v}}^{2}\right]$

In our implementation for NGC 5548, we have chosen the velocities $v_{i}$ of the velocity components as we discussed at the end of Sect. 4.3 (i.e. five components at outflow velocities of $-163,-305,-375,-540$ and $\left.-669 \mathrm{~km} \mathrm{~s}^{-1}\right)$. We also took $\tau_{i}=\tau_{0} / 5$ for all these components, with $\tau_{0}$ the total optical depth given by

$\tau_{0}=0.106 f N_{20} \lambda / \sigma_{\mathrm{v}, 100}$.

Here $f$ is the oscillator strength, $\lambda$ the wavelength in $\AA, \sigma_{\mathrm{v}, 100}$ the velocity dispersion in units of $100 \mathrm{~km} \mathrm{~s}^{-1}$ and $N_{20}$ the total column density of the ion in units of $10^{20} \mathrm{~m}^{-2}$, added for all five components.

We applied this absorber model to the continuum model described before and fitted the entire spectrum. In addition, we have included in the model the strongest narrow emission components for the $\mathrm{O}$ viI triplet and the Ne IX forbidden line as found by Kaastra et al. (2000).

In a more general implementation of this model (not used here for NGC 5548) we take instead

$v_{i}=v_{0}+i \Delta v$,

$\tau_{i}=K \exp \left[-v_{i}^{2} / 2 b^{2}\right]$,

where $v_{0}$ is the average velocity of the blend (a negative value corresponds to a blue-shift or outflow), $\Delta v$ is the separation between the components, and the rms width of the blend $b$ is in general larger than the intrinsic width $\sigma_{\mathrm{v}}$ of the components. The normalization $K$ is defined in such a way that $\sum \tau_{i}=\tau_{0}$. 
Table 4. Fits to the total spectrum.

\begin{tabular}{|c|c|}
\hline Parameter & value \\
\hline$\chi^{2}$ & 2211 \\
\hline degrees of freedom & 1963 \\
\hline Power law photon index $\Gamma$ & $1.848 \pm 0.021$ \\
\hline Power law normalization ${ }^{\mathrm{a}}$ & $131.8 \pm 1.6$ \\
\hline MBB Temperature (keV) & $0.081 \pm 0.007$ \\
\hline MBB Normalization ${ }^{\mathrm{b}}$ & $(2.3 \pm 0.6) \times 10^{33}$ \\
\hline C VI Ly $\alpha^{\mathrm{c}} 33.736 \AA$ & $0(<8)$ \\
\hline N VII Ly $\alpha^{\mathrm{c}} 24.781 \AA$ & $11.6 \pm 4.0$ \\
\hline O VIII Ly $\alpha^{\mathrm{c}} 18.969 \AA$ & $6.5 \pm 2.3$ \\
\hline C VI Ly $\alpha^{\mathrm{d}} 33.736 \AA$ & $<0.7$ \\
\hline N VII Ly $\alpha^{\mathrm{d}} 24.781 \AA$ & 1.1 \\
\hline O VIII Ly $\alpha^{\mathrm{d}} 18.969 \AA$ & 0.6 \\
\hline Inclination $i$ (degrees) & 46.0 \\
\hline Inner radius $r_{1} \mathrm{e}$ & 1.6 \\
\hline Outer radius $r_{2}{ }^{\mathrm{e}}$ & 40 \\
\hline Scale height $h^{\mathrm{e}}$ & 0.0 \\
\hline Emissivity index $q$ (fixed) & 3.9 \\
\hline Narrow emission lines & see Tables 8 and 9 \\
\hline Column densities & see Table 5 \\
\hline
\end{tabular}

a In photons $\mathrm{m}^{-2} \mathrm{~s}^{-1} \mathrm{keV}^{-1}$ at $1 \mathrm{keV}$.

b Emitting area times square root of electron density in $m^{0.5}$.

c Observed line intensities in units of $\mathrm{ph} \mathrm{m}^{-2} \mathrm{~s}^{-1}$ corrected for Galactic absorption.

d Equivalent width in $\AA$.

e In units of $G M / c^{2}$; parameters fixed.

\subsection{Fit results using the slab model}

The results of our fit are summarized in Tables 4 and 5 . We have kept the shape parameters of the relativistically broadened line profiles for Ly $\alpha$ of O VIII, N VII and C VI fixed to the values given in Table 1, given their large uncertainties. However the strength of the lines was allowed to vary. The intrinsic width of the absorption line components was kept fixed to $\sigma_{\mathrm{v}}=32 \mathrm{kms}^{-1}$ (rms width), in agreement with the UV line components. When we left it as a free parameter, the best fit parameter was $47_{-12}^{+59} \mathrm{~km} \mathrm{~s}^{-1}$, in fair agreement with our fixed value.

In those cases where a zero column density is within the error bars, we put the lower error on the logarithm of the column density formally to $-\infty$. The measured column densities agree in general within their error bars with the values derived from the strongest individual lines as given by Kaastra et al. (2000). For completeness, we also include the total column densities for the co-added components $2-5$ for $\mathrm{HI}, \mathrm{C}$ IV, $\mathrm{N} \mathrm{V}$ and $\mathrm{OVI}$ as derived from UV measurements by the Space Telescope Imaging Spectrograph (STIS) as well as the Far Ultraviolet Spectroscopic Explorer (FUSE). We comment on these UV lines in Sect. 5.4.

In Figs. 8-16 we show the best fit spectrum with the transmission of the model components included.

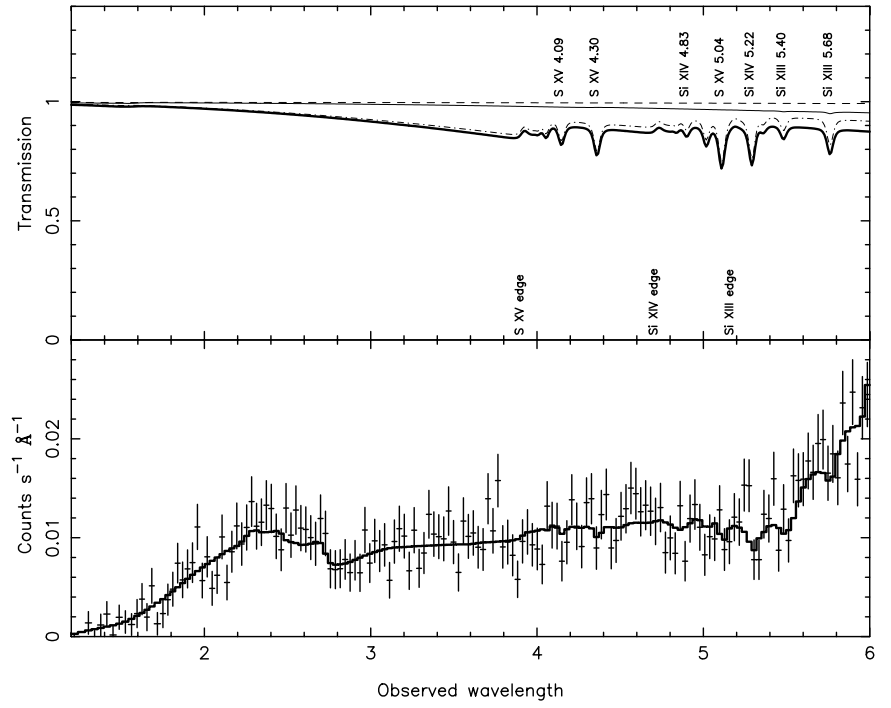

Fig. 8. lower panel: observed spectrum with the best fit model. Upper panel: transmission of the high ionization absorber component A (dash-dotted line), medium ionization component B (thin solid line), low ionization component $\mathrm{C}$ (dashed line) and total transmission (thick solid line). Line identifications are given with their rest frame wavelengths.

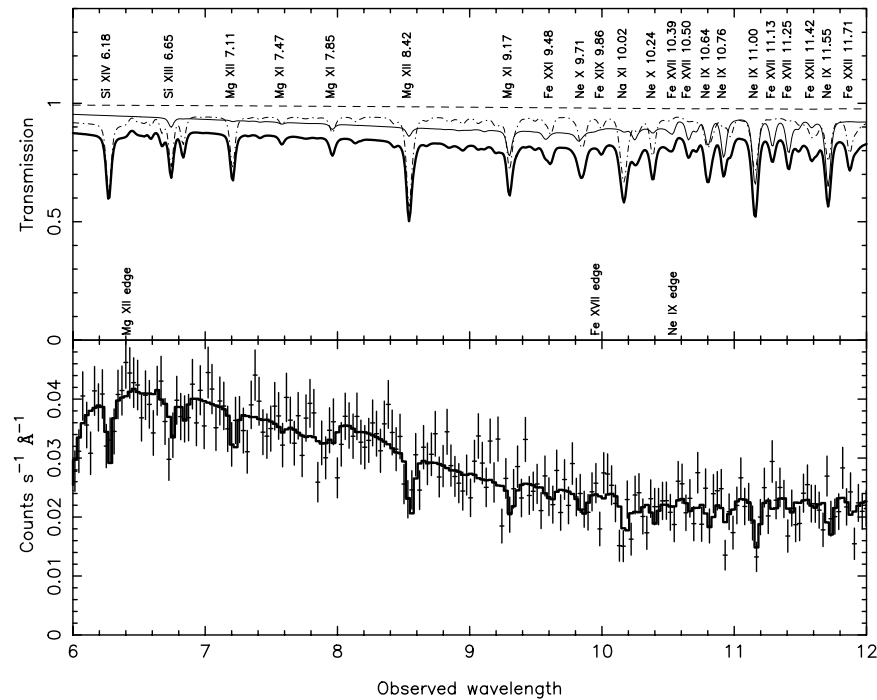

Fig. 9. As Fig. 8, but for 6-12 $\AA$.

\subsection{Other atomic data for inner-shell transitions}

Wavelengths and oscillator strengths for the $2 \mathrm{p}-3 \mathrm{~d}$ UTA of Fe I-Fe XVI have also been calculated recently by Behar et al. (2001b). Their results are in good agreement with the present calculations for these ions. We have tested this explicitly by using the Behar et al. data in our spectral fits; the derived column densities are all within the error bars consistent with the results presented in Table 5 .

Recently it has also become evident that inner shell transitions of other ions such as oxygen may be important in AGN (Behar \& Netzer 2002). In our current modeling, we have taken the O VI data from Pradhan (2000). From Table 5 we see that the column density of this ion is poorly 
Table 5. Measured and model column densities. Listed are the logarithms of the column densities in units of $\mathrm{m}^{-2}$. The columns labeled A, B and C list as a percentage the relative contribution of the three ionization components to the total model column density. The last three columns give rest frame wavelength, oscillator strength and optical depth at line center for the strongest line of each ion in the LETGS or UV band, for $\sigma_{\mathrm{v}}=32 \mathrm{~km} \mathrm{~s}^{-1}$ and a single velocity component with $20 \%$ of the total (observed) ion column density.

\begin{tabular}{|c|c|c|c|c|c|c|c|c|}
\hline Ion & $\mathrm{A}$ & $\mathrm{B}$ & $\mathrm{C}$ & model & observed & $\lambda(\AA)$ & $f$ & $\overline{\tau_{0}}$ \\
\hline H I & 18 & 45 & 38 & 19.65 & $19.65(-0.02,+0.02)$ & 1215.670 & 0.42 & 15.0 \\
\hline C IV & 0 & 1 & 99 & 19.02 & $19.08(-0.03,+0.03)$ & 1548.195 & 0.19 & 2.3 \\
\hline $\mathrm{C} \mathrm{V}$ & 1 & 44 & 55 & 20.40 & $20.56(-0.38,+0.30)$ & 40.268 & 0.65 & 6.3 \\
\hline $\mathrm{C} \mathrm{VI}$ & 43 & 55 & 1 & 21.70 & $21.32(-0.32,+0.29)$ & 33.736 & 0.42 & 19.4 \\
\hline $\mathrm{N}$ V & 0 & 7 & 93 & 18.93 & $19.11(-0.03,+0.03)$ & 1238.820 & 0.16 & 1.7 \\
\hline N VI & 3 & 81 & 16 & 20.10 & $20.19(-0.39,+0.34)$ & 28.787 & 0.68 & 2.0 \\
\hline N VII & 53 & 47 & 0 & 21.25 & $20.81(-0.25,+0.29)$ & 24.781 & 0.42 & 4.4 \\
\hline \multirow[t]{2}{*}{$\mathrm{O} \mathrm{VI}$} & 0 & 36 & 64 & 19.98 & $19.61(-0.13,+0.13)$ & 1031.926 & 0.13 & 3.7 \\
\hline & & & & & $19.90(-\infty,+0.63)$ & 22.007 & 0.52 & 0.3 \\
\hline O VII & 7 & 91 & 3 & 21.25 & $21.61(-0.17,+0.14)$ & 21.602 & 0.70 & 40.6 \\
\hline O VIII & 67 & 33 & 0 & 22.17 & $22.21(-0.15,+0.11)$ & 18.969 & 0.42 & 84.8 \\
\hline Ne IX & 25 & 75 & 0 & 21.38 & $21.66(-0.31,+0.19)$ & 13.447 & 0.72 & 29.5 \\
\hline $\mathrm{Ne} \mathrm{X}$ & 91 & 9 & 0 & 22.13 & $21.44(-0.32,+0.30)$ & 12.134 & 0.42 & 9.2 \\
\hline $\mathrm{Na} \mathrm{X}$ & 49 & 51 & 0 & 20.29 & $20.48(-\infty,+0.64)$ & 11.003 & 0.73 & 1.6 \\
\hline $\mathrm{Na} X I$ & 97 & 3 & 0 & 21.03 & $20.92(-0.66,+0.48)$ & 10.025 & 0.42 & 2.3 \\
\hline Mg IX & 0 & 98 & 1 & 20.37 & $20.30(-0.44,+0.28)$ & 62.751 & 0.54 & 4.5 \\
\hline $\operatorname{Mg} X$ & 7 & 93 & 0 & 20.47 & $18.15(-\infty,+1.74)$ & 57.876 & 0.22 & 0.0 \\
\hline $\mathrm{Mg}$ XI & 74 & 26 & 0 & 21.29 & $20.95(-1.23,+0.70)$ & 9.169 & 0.74 & 4.0 \\
\hline Mg XII & 99 & 1 & 0 & 21.93 & $22.08(-0.48,+0.25)$ & 8.421 & 0.42 & 27.9 \\
\hline Si VIII & 0 & 14 & 86 & 18.78 & $19.62(-1.02,+0.45)$ & 61.070 & 0.62 & 1.0 \\
\hline Si IX & 0 & 81 & 19 & 19.22 & $20.26(-0.57,+0.43)$ & 55.305 & 0.95 & 6.3 \\
\hline Si X & 1 & 98 & 1 & 20.19 & $20.04(-0.34,+0.23)$ & 47.489 & 0.21 & 0.7 \\
\hline Si XI & 5 & 95 & 0 & 20.55 & $19.74(-0.45,+0.29)$ & 43.762 & 0.48 & 0.8 \\
\hline Si XII & 47 & 53 & 0 & 20.52 & $14.57(-\infty,+5.83)$ & 31.012 & 0.06 & 0.0 \\
\hline Si XIII & 97 & 3 & 0 & 21.54 & $21.59(-0.79,+0.54)$ & 6.648 & 0.76 & 13.0 \\
\hline Si XIV & 100 & 0 & 0 & 21.84 & $22.19(-0.85,+0.36)$ & 6.182 & 0.42 & 26.4 \\
\hline S X & 1 & 89 & 11 & 19.62 & $19.95(-0.36,+0.33)$ & 42.543 & 0.67 & 1.7 \\
\hline S XI & 3 & 97 & 0 & 20.31 & $20.29(-0.60,+0.41)$ & 39.240 & 1.05 & 5.3 \\
\hline S XII & 13 & 87 & 0 & 20.59 & $19.99(-0.48,+0.33)$ & 36.398 & 0.62 & 1.5 \\
\hline S XIII & 43 & 57 & 0 & 20.80 & $13.88(-\infty,+5.56)$ & 32.242 & 0.45 & 0.0 \\
\hline S XIV & 91 & 9 & 0 & 21.06 & $14.89(-\infty,+5.06)$ & 23.015 & 0.30 & 0.0 \\
\hline S XV & 100 & 0 & 0 & 21.95 & $22.19(-0.65,+0.28)$ & 5.039 & 0.77 & 39.7 \\
\hline Ar XIII & 12 & 88 & 0 & 19.57 & $14.41(-\infty,+4.41)$ & 29.320 & 0.91 & 0.0 \\
\hline Ar XIV & 43 & 57 & 0 & 19.65 & $14.30(-\infty,+5.18)$ & 27.470 & 0.65 & 0.0 \\
\hline Ar XV & 84 & 16 & 0 & 19.86 & $20.00(-\infty,+0.54)$ & 24.737 & 0.39 & 0.6 \\
\hline Fe VII & 0 & 0 & 100 & 19.18 & $19.37(-\infty,+0.73)$ & 16.903 & 0.18 & 0.0 \\
\hline Fe VIII & 0 & 0 & 100 & 19.87 & $19.10(-\infty,+0.86)$ & 16.658 & 0.36 & 0.1 \\
\hline Fe IX & 0 & 0 & 100 & 20.15 & $18.48(-\infty,+1.52)$ & 16.510 & 1.41 & 0.0 \\
\hline Fe X & 0 & 0 & 100 & 20.28 & $19.68(-\infty,+0.43)$ & 16.339 & 0.76 & 0.4 \\
\hline Fe XI & 0 & 0 & 100 & 20.14 & $20.27(-0.19,+0.15)$ & 16.132 & 0.34 & 0.7 \\
\hline Fe XII & 0 & 14 & 86 & 19.92 & $19.80(-\infty,+0.37)$ & 15.963 & 0.42 & 0.3 \\
\hline Fe XIII & 0 & 87 & 13 & 20.23 & $19.35(-\infty,+0.53)$ & 15.849 & 0.70 & 0.2 \\
\hline Fe XIV & 0 & 98 & 2 & 20.31 & $18.94(-\infty,+0.70)$ & 15.579 & 0.61 & 0.1 \\
\hline Fe XV & 0 & 100 & 0 & 20.57 & $19.78(-0.60,+0.38)$ & 15.316 & 1.74 & 1.1 \\
\hline Fe XVI & 0 & 100 & 0 & 20.17 & $20.47(-0.44,+0.29)$ & 15.250 & 0.96 & 2.9 \\
\hline Fe XVII & 2 & 98 & 0 & 20.64 & $20.95(-0.21,+0.18)$ & 15.014 & 2.72 & 24.1 \\
\hline Fe XVIII & 36 & 64 & 0 & 20.42 & $20.14(-0.39,+0.27)$ & 14.204 & 0.88 & 1.1 \\
\hline Fe XIX & 91 & 9 & 0 & 20.52 & $20.69(-0.19,+0.16)$ & 13.521 & 0.72 & 3.1 \\
\hline Fe XX & 99 & 1 & 0 & 20.79 & $20.64(-0.25,+0.21)$ & 12.827 & 0.47 & 1.8 \\
\hline Fe XXI & 100 & 0 & 0 & 20.57 & $20.29(-0.67,+0.43)$ & 12.286 & 1.24 & 2.0 \\
\hline Fe XXII & 100 & 0 & 0 & 20.31 & $20.27(-\infty,+0.45)$ & 11.706 & 0.67 & 1.0 \\
\hline Fe XXIII & 100 & 0 & 0 & 19.96 & $20.10(-\infty,+0.55)$ & 10.981 & 0.41 & 0.4 \\
\hline Fe XXIV & 100 & 0 & 0 & 19.23 & $15.00(-\infty,+4.72)$ & 10.620 & 0.24 & 0.0 \\
\hline
\end{tabular}




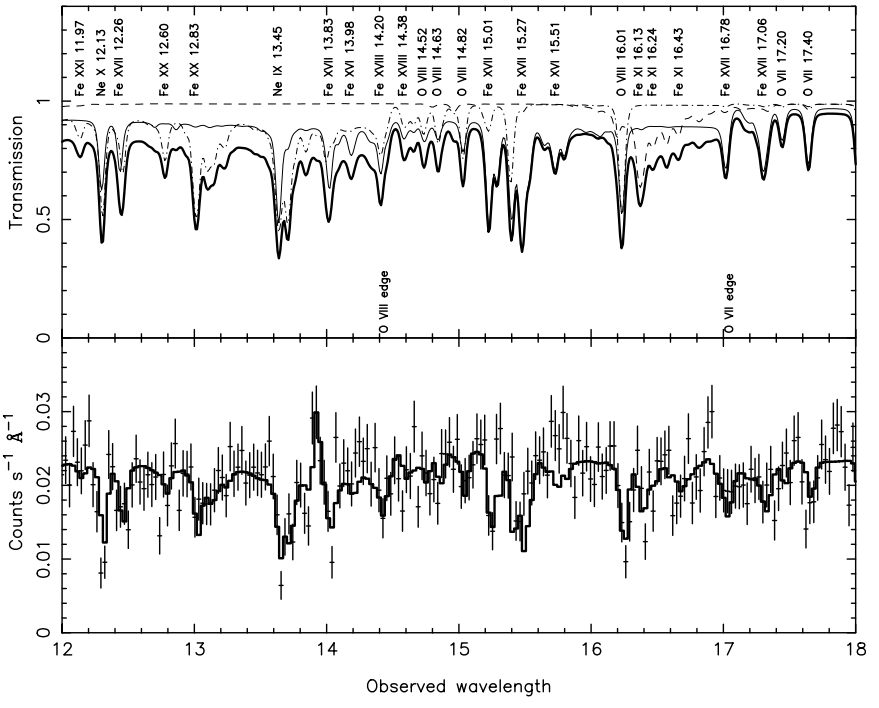

Fig. 10. As Fig. 8, but for $12-18 \AA$.

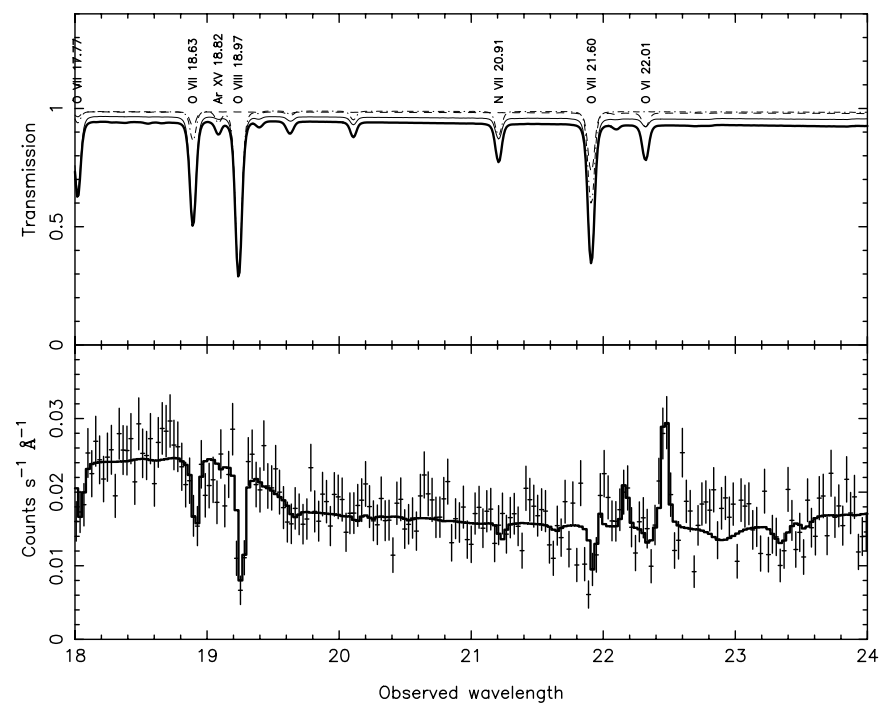

Fig. 11. As Fig. 8, but for 18-24 A.

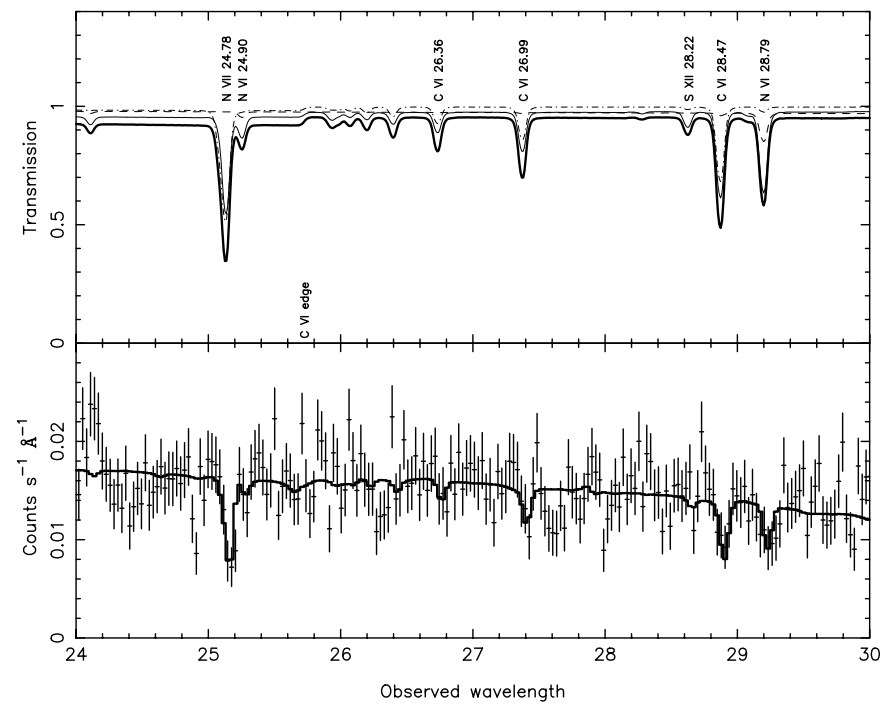

Fig. 12. As Fig. 8, but for $24-30 \AA$.

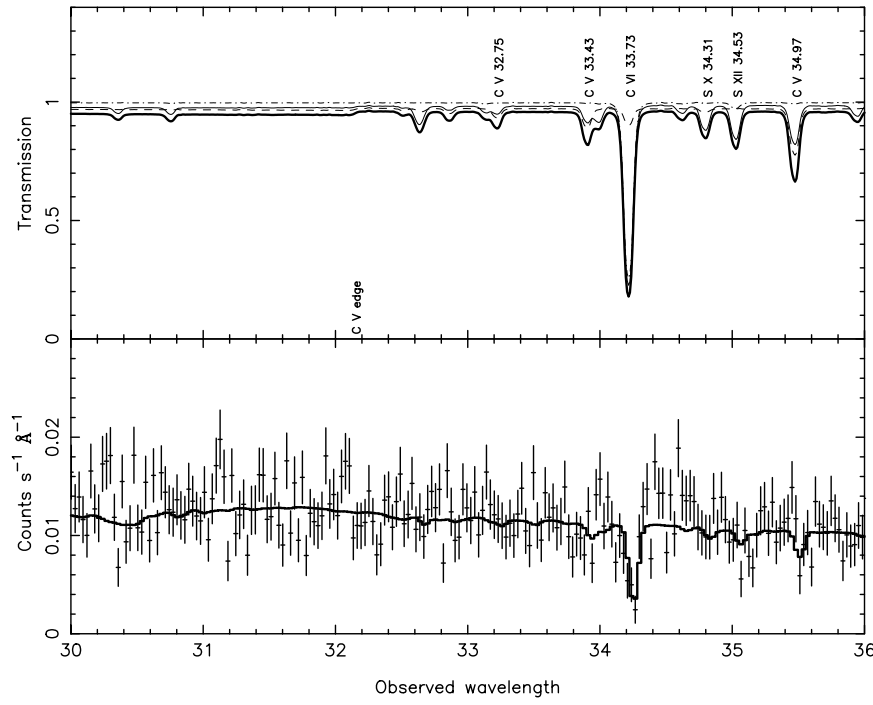

Fig. 13. As Fig. 8, but for $30-36 \AA$.

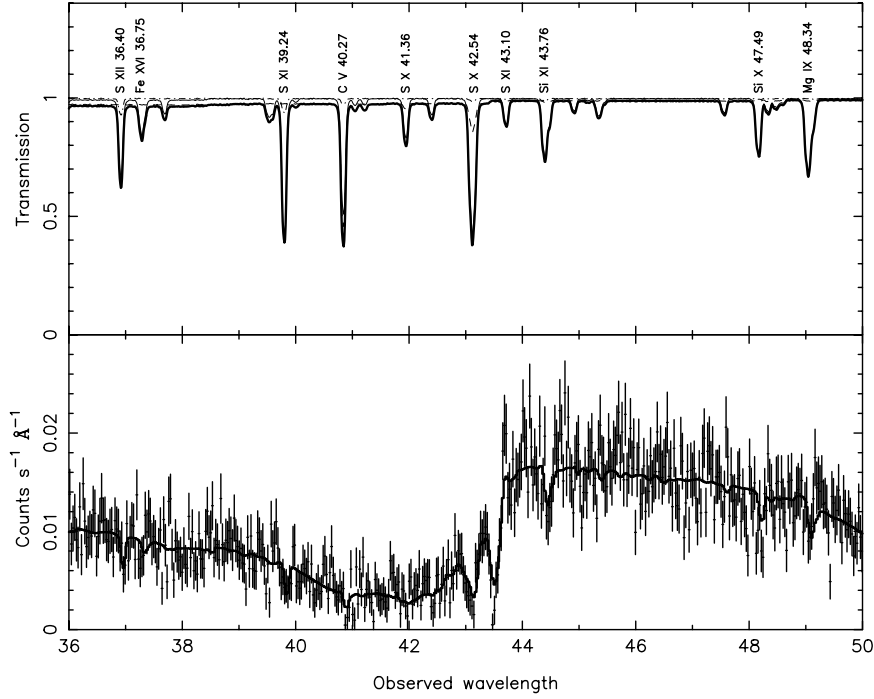

Fig. 14. As Fig. 8, but for $36-50 \AA$.

constrained. The wavelengths and oscillator strengths for all of the inner shell $1 \mathrm{~s}-n \mathrm{p}(n \leq 7)$ transitions in $\mathrm{O}$ I-O VI have been recently calculated (Behar 2002, in preparation). Lines from these ions have recently been discovered in the RGS spectrum of NGC 5548 (Steenbrugge et al. 2002). We included those lines also in our code but did not detect significant absorption from these ions (Table 6). Therefore we did not take these ions into account in our further analysis, except for O vi but with the value quoted in Table 5.

\subsection{Supplementary UV data}

The column densities that we derived can be compared to the predictions from photoionization models. Before this comparison we want to supplement the data with UV data, which can help to constrain the lowest ionization components. 


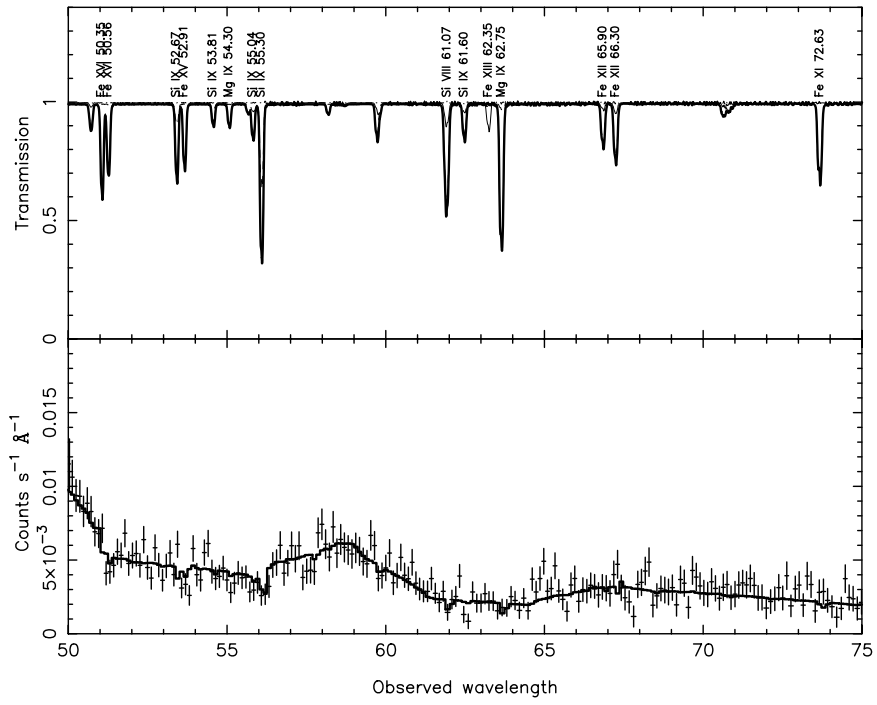

Fig. 15. As Fig. 8, but for $50-75 \AA$.

Table 6. $1 \sigma$ upper limits to the oxygen column densities. Listed are the logarithms of the column densities in units of $\mathrm{m}^{-2}$.

\begin{tabular}{lr}
\hline \hline Ion & observed \\
\hline O I & $<20.13$ \\
O II & $<20.06$ \\
O III & $<20.68$ \\
O IV & $<19.68$ \\
O V & $<20.48$ \\
O VI & $<20.44$ \\
\hline
\end{tabular}

For CIV, it was shown recently by Arav et al. (2002) that the column density as derived by Crenshaw \& Kraemer (1999) is four times too small. This is due in part to the fact that apparently the narrow line region is not covered by the warm absorber. Also the fact that the line is highly saturated, so that the line profile merely represents the covering factor and not the optical depth of the absorbing gas, contributes to this. Thus, we adopted the column density derived by Arav et al.

For H I, Crenshaw \& Kraemer obtain a logarithmic column of $18.80 \pm 0.02$, based upon the Ly $\alpha$ line as observed with the STIS in March 1998. Recently, Brotherton et al. (2002) determined a H I column density of $19.65 \pm 0.02$, based upon the Ly $\beta$ line observed by FUSE in June 2000. If not caused by strong variability, this last column density would imply that the Ly $\alpha$ line, with its higher oscillator strength, should have an even higher optical depth than the C IV line. Hence we may expect similar saturation problems for Ly $\alpha$ as for C IV. Therefore we prefer the column density derived from the $\operatorname{Ly} \beta$ line.

Optical depth effects are estimated to be less important for $\mathrm{N} \mathrm{v}$, so for that ion we adopt the STIS values of Crenshaw \& Kraemer (1999).

Finally, our estimate for the O vi column density, based upon the present X-ray data, is rather uncertain: the best fit logarithmic column density is $19.90_{-\infty}^{+0.63}$. This value is,

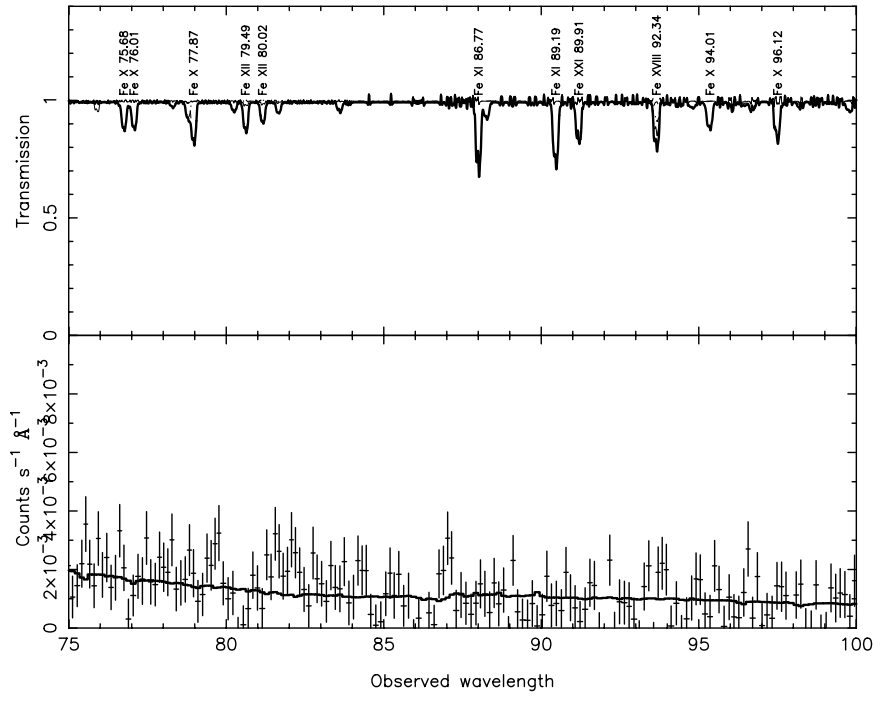

Fig. 16. As Fig. 8, but for $75-100 \AA$.

however, consistent with the column density derived from the ultraviolet 1032, $1038 \AA \AA$ lines: $19.61 \pm 0.13$ (Brotherton et al. 2002). We increased the uncertainty in this number to take into account the effects of incomplete covering of the central source. We used the latter more accurate value in all subsequent modeling.

\subsection{Modeling of the column density}

The column densities that we derived can be compared to the values predicted by photoionization calculations. As in Kaastra et al. (2000) we have chosen to compare the column densities with calculations made with the XSTAR code (Kallman \& Krolik 1999). The main difference with the previous work is the larger amount of ions that is available now, in particular the long-wavelength L-shell ions of $\mathrm{Mg}, \mathrm{Si}, \mathrm{S}$ and $\mathrm{Ar}$ as well as the inner-shell UTA transitions of M-shell iron ions.

We produced a grid of models using the following ionizing spectrum. For $\lambda>80 \AA$ we used the parameterization of Mathur et al. (1995) and below $80 \AA$ we used the continuum as derived from the present fit to the Chandra data, with an exponential high-energy cut-off at $115 \mathrm{keV}$ as found before using BeppoSAX observations of this source (Nicastro et al. 2000). The 1-1000 Ryd flux of the model is $3.10 \times 10^{-13} \mathrm{~W} \mathrm{~m}^{-2}$, corresponding to an intrinsic source luminosity of $3.78 \times 10^{37} \mathrm{~W}$ in the same energy band. We fixed the gas density to $10^{14} \mathrm{~m}^{-3}$, but the model is not very sensitive to this. Abundances were fixed to solar values (Anders \& Grevesse 1989), and we used small column densities in order to prevent strong variations of the ion concentrations within the slab. The ionization parameter $\xi \equiv L / n r^{2}$ is expressed in the usual units of $10^{-9} \mathrm{Wm}$ $\left(1 \mathrm{erg} \mathrm{s}^{-1} \mathrm{~cm}\right)$ throughout this paper. Here $L$ is the ionizing luminosity in the 1-1000 Ryd band, $n$ the gas density and $r$ the distance of the slab from the ionizing source.

We calculated models for $\log \xi$ ranging from 0.4 to 4.0 in steps of 0.1. The output of the model consists of a set 
Table 7. Total column densities, ionization parameters and abundances for the three components A, B and C. The listed abundances are the logarithms of the abundances in solar units.

\begin{tabular}{|c|c|c|c|}
\hline Parameter & $\mathrm{A}$ & $\mathrm{B}$ & $\mathrm{C}$ \\
\hline $\log N_{\mathrm{H}}\left(\mathrm{m}^{-2}\right)$ & $26.93 \pm 0.20$ & $25.67 \pm 0.17$ & $23.88 \pm 0.13$ \\
\hline $\log \xi$ & $2.87 \pm 0.15$ & $1.87 \pm 0.17$ & $0.50 \pm 0.20$ \\
\hline$T(\mathrm{eV})$ & $43 \pm 8$ & $8.0 \pm 2.4$ & $2.0 \pm 0.2$ \\
\hline \multicolumn{4}{|l|}{ Abundances: } \\
\hline $\mathrm{C}$ & & $-0.08 \pm 0.12$ & \\
\hline $\mathrm{N}$ & & $-0.32 \pm 0.15$ & \\
\hline $\mathrm{O}$ & & $-0.56 \pm 0.07$ & \\
\hline $\mathrm{Ne}$ & & $-0.19 \pm 0.16$ & \\
\hline $\mathrm{Na}$ & & $0.33 \pm 0.38$ & \\
\hline $\mathrm{Mg}$ & & $-0.17 \pm 0.18$ & \\
\hline $\mathrm{Si}$ & & $-0.32 \pm 0.14$ & \\
\hline S & & $0.03 \pm 0.16$ & \\
\hline $\mathrm{Ar}$ & & $-0.21 \pm 0.53$ & \\
\hline $\mathrm{Fe}$ & $-1.18 \pm 0.43$ & $0.02 \pm 0.07$ & $1.45 \pm 0.56$ \\
\hline
\end{tabular}

of ionic column densities. With these we tried to explain the observed column densities as a linear combination of a minimal number of ionization components. A single component is not sufficient to explain the broad range of ions. We need at least two additional ionization components. We label these components $\mathrm{A}, \mathrm{B}$ and $\mathrm{C}$ with decreasing ionization parameter $\xi$.

The dominant ionization component (B) produces amongst others the strong $\mathrm{O}$ VII absorption lines, and was first discovered by Kaastra et al. (2000). In addition, we find evidence for a second, less ionized component (C). This mainly produces the UV lines, as well as C V, Si VIII lines and the UTA of Fe VII-Fe XII. Finally, a very high ionization component (A) producing most of the hydrogenic ions as well as the L-shell transitions of Fe XIX-Fe XXIV is also found.

In Table 5 we list in addition to the observed column densities our best-fit total column densities, as well as the percentage contribution of each of the three components. The best fit ionization parameters, column densities and abundances for each component are listed in Table 7 . The temperatures listed in this table are not free fit parameters, but are derived from the XSTAR models. In our fit, we have assumed that the abundances are the same in each component, except for iron, where we allowed the abundance of each component to vary independently. In fact, we could not obtain a good fit if we forced the iron abundance to be the same. We will discuss these results further in Sect. 6.4.

\subsection{Narrow emission lines}

Only a limited number of narrow emission lines is detected significantly in the X-ray spectrum of NGC 5548. These lines are listed in Tables 8 and 9 . The intercombination $(i)$ and forbidden $(f)$ lines of the He-like O VII and Ne IX have a very small oscillator strength, and therefore no sig-

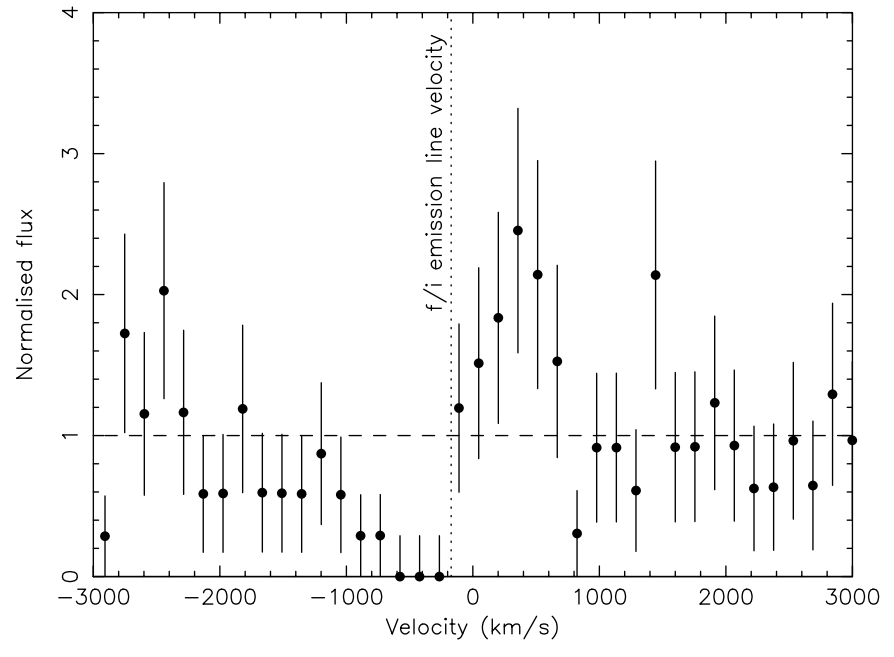

Fig. 17. MEG spectrum around the O vin Ly $\alpha$ line. Bin size is $0.01 \AA$, negative velocities indicate blue-shift. The spectrum has been normalized to 1 for the continuum. The average velocity of the forbidden and intercombination lines of $\mathrm{O}$ VII and Ne IX is also indicated.

Table 8. Narrow emission lines fluxes. Unit: photons $\mathrm{m}^{-2} \mathrm{~s}^{-1}$, corrected for Galactic absorption. Wavelengths are the rest frame wavelengths in $\AA$.

\begin{tabular}{lrrr}
\hline \hline line & $\lambda$ & LETGS & MEG \\
\hline O viII Ly $\alpha$ & 18.969 & $0.16 \pm 0.12$ & $0.19 \pm 0.10$ \\
O viI r & 21.602 & $0.30 \pm 0.16$ & $0.15 \pm 0.15$ \\
O viI i & 21.804 & $0.27 \pm 0.17$ & $0.20 \pm 0.15$ \\
O viI f & 22.101 & $0.81 \pm 0.19$ & $0.82 \pm 0.26$ \\
Ne Ix f & 13.700 & $0.25 \pm 0.10$ & $0.09 \pm 0.04$ \\
\hline
\end{tabular}

Table 9. Velocities of the narrow emission lines, rounded to multiples of $10 \mathrm{~km} \mathrm{~s}^{-1}$. The last column gives the MEG values corrected for a velocity of $-172 \mathrm{~km} \mathrm{~s}^{-1}$ as described in the text.

\begin{tabular}{lrrr}
\hline \hline line & LETGS & MEG & MEG (corr.) \\
\hline O viII Ly $\alpha$ & $+650 \pm 550$ & $+240 \pm 110$ & +420 \\
O vil r & $+680 \pm 240$ & $+170 \pm 180$ & +340 \\
O viI i & $+10 \pm 330$ & $-120 \pm 140$ & +50 \\
O viI f & $-70 \pm 100$ & $-180 \pm 50$ & -0 \\
Ne IX f & $-330 \pm 260$ & $-200 \pm 130$ & -30 \\
\hline
\end{tabular}

nificant absorption component. This makes it easier to measure the intensity and average velocity than for the resonance lines. The O VII resonance line $(r)$ as well as the $\mathrm{O}$ VIII Ly $\alpha$ line have a strong absorption component. For the LETGS data, the uncertainties on the average velocities include the systematic uncertainties of Fig. 1, which are much smaller than the statistical errors. The Chandra proposers Guide lists a relative wavelength accuracy for the MEG data of $0.0055 \AA$, and $0.011 \AA$ in absolute value. At $22 \AA$, this corresponds to an uncertainty of 75 and $150 \mathrm{kms}^{-1}$, respectively. From Table 9 we see 


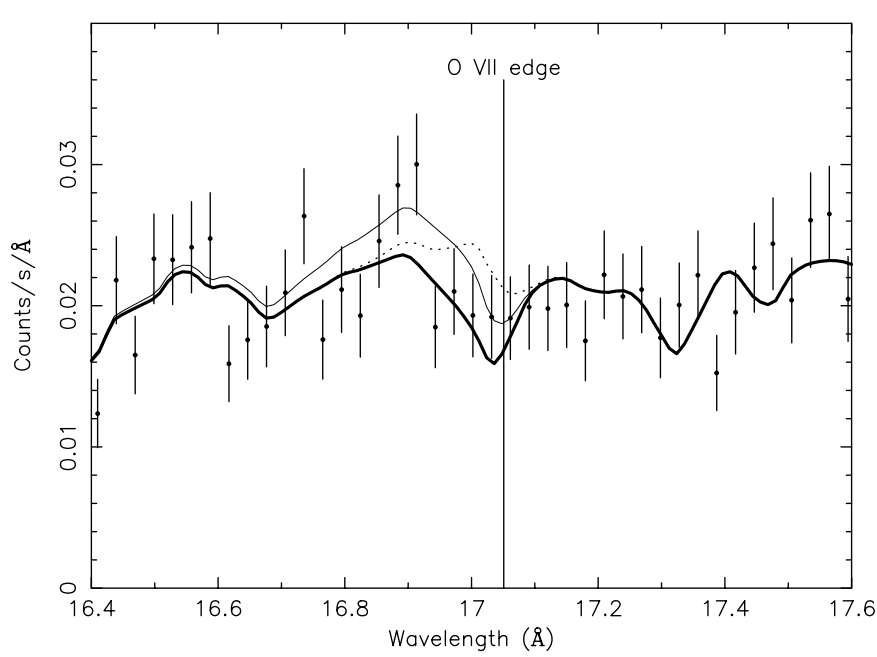

Fig. 18. The spectrum near $17 \AA$ with the best fit model of Fig. 10 only, (thick solid line) or with the O viI RRC included for a temperature of $2 \mathrm{eV}$ (dotted line) and $8 \mathrm{eV}$ (thin solid line). The flux of the RRC was derived from the forbidden line of O viI.

that the intercombination and forbidden lines as measured with the MEG are all blue-shifted, with an average value of $-172 \pm 47 \mathrm{~km} \mathrm{~s}^{-1}$. This is close to the systematic uncertainty, and significantly different from the centroids as measured with the LETGS. In the last column of Table 9 we have given the line centroids with this constant offset of $-172 \mathrm{~km} \mathrm{~s}^{-1}$ subtracted. With this correction, both wavelength scales agree within $120 \mathrm{~km} \mathrm{~s}^{-1}$.

\subsection{Radiative recombination continua}

NGC 5548 produces mostly strong X-ray absorption lines. The strongest emission lines visible in the spectrum are the forbidden and intercombination lines of He-like oxygen and neon. These lines are mostly formed through recombination under the conditions that prevail in NGC 5548. The upper levels of these lines are populated by radiative cascades following radiative recombination from shells above $n=2$, as well as by direct recombination into the $n=2$ shell. A recombining plasma in photoionization equilibrium should also yield a significant number of recombinations directly to the ground state. We thus expect significant Radiative Recombination Continua (RRC), which due to the prevailing low temperatures should have a narrow width.

The most promising candidate appears to be the O VII $\mathrm{RRC}$, given the strong forbidden line, as well as the large column density of its parent ion O viII. We could not detect a significant narrow RRC at the expected wavelength of $16.77 \AA$, and its best-fit absorption-corrected flux is $0.06 \pm 0.10$ photons $\mathrm{m}^{-2} \mathrm{~s}^{-1}$. Comparing this with the strength of the forbidden line from Table 8 we find a ratio $\mathrm{RRC} / f$ of $0.07 \pm 0.12$. This is much smaller than predicted by recombination models. For example, Behar et al. (2001a) in their modeling for the Seyfert 2 galaxy

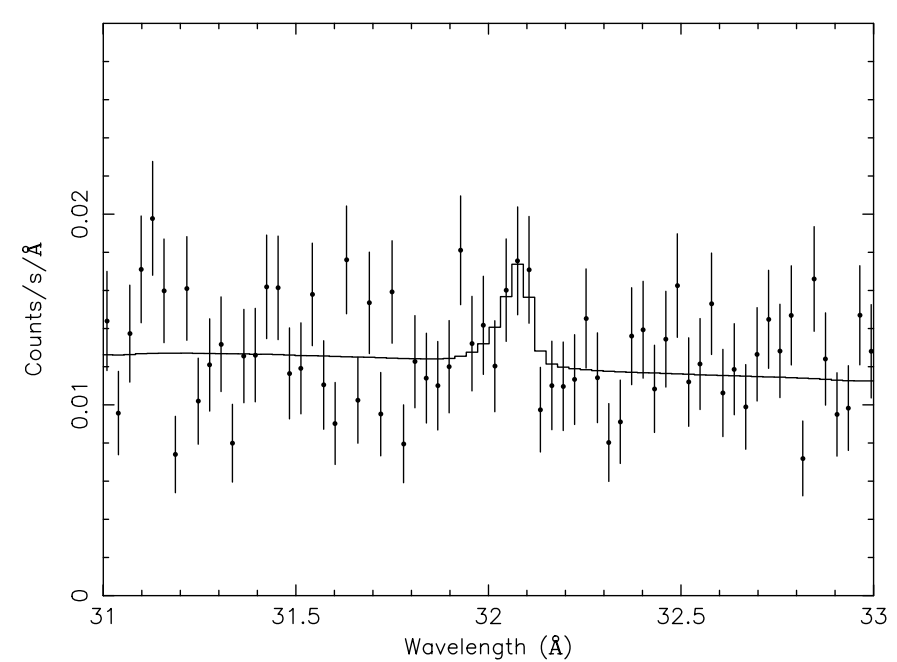

Fig. 19. The best-fit $\mathrm{CV}_{\mathrm{V}} \mathrm{RRC}$ superimposed upon the local continuum.

NGC 1068 find a ratio RRC/ $f$ of 0.57 for an adopted temperature of $5 \mathrm{eV}$. The temperature dependence on this ratio is weak. Also in the MEG data this ratio is small: $0.22 \pm 0.25$. Thus, there appears to be a problem with the predicted strength of the O vII RRC.

But are we really missing the RRC? We calculated the flux and spectrum of the RRC based upon the O VII forbidden line as a function of temperature (Fig. 18). We see from this figure why a low temperature RRC does not fit: it over-predicts the flux around $17.0 \AA$. However for a higher temperature of $8 \mathrm{eV}$ a better match is obtained, in particular near $16.9 \AA$. On the other hand, there is now a $\sim 2 \sigma$ flux deficit near $16.95 \AA$. This corresponds to a rest frame wavelength of $16.67 \AA$ and coincides with the strongest inner shell transition of Fe VIII (see also Table 5). Thus the dip can be compensated for in principle by slightly increasing the column density of Fe VIII. It is evident that the situation near this wavelength is rather complex. This is even more the case given the fact that one of the strong Fe XVII lines at $16.78 \AA$ is also within $0.01 \AA$ or $200 \mathrm{~km} \mathrm{~s}^{-1}$ from the O vII edge. This line has an estimated optical depth at line center of 1.0 for each velocity component. Therefore it will interfere with the RRC (in fact, the dip near $17.05 \AA$ in the original model of Fig. 18 is due to this Fe XVII line).

Given this and the limited statistics of our data further hard conclusions are difficult to obtain. We conclude that the data are not inconsistent with RRC emission of a temperature of $8 \mathrm{eV}$, the predicted temperature of the O VII ions based upon our XSTAR modeling.

Another important $\mathrm{RRC}$ is that of $\mathrm{CV}$. There is a clear indication for the presence of this RRC in the spectrum (see Fig. 19). We find an absorption-corrected flux of $0.47_{-0.24}^{+0.45}$ photons $\mathrm{m}^{-2} \mathrm{~s}^{-1}$, corresponding to an emission measure $n_{\mathrm{e}} n_{\mathrm{CVI}} V$ of $9.5 \times 10^{66} \mathrm{~m}^{-3}$, where $n_{\mathrm{e}}$ is the electron density, $n_{\mathrm{C} \text { VI }}$ the density of the parent ion $\mathrm{C}$ VI and $V$ the emitting volume. For the temperature we obtain only an upper limit: the best-fit value is $0.5 \pm 0.5 \mathrm{eV}$, 
which is smaller than the modeled temperature of ionization component $\mathrm{C}(2.0 \mathrm{eV})$. The corresponding forbidden line of $\mathrm{C}$ V at $41.47 \AA$ is difficult to measure, because it is redshifted into the deep neutral carbon edge region of the LETGS instrument. Formally, we find a best-fit absorption corrected flux of $0.26 \pm 0.34$ photons $\mathrm{m}^{-2} \mathrm{~s}^{-1}$, but the systematic uncertainty is likely to be as large as the statistical error. Hence, the $\mathrm{RRC} / f$ ratio for $\mathrm{C} v$ remains inconclusive.

The measured blue-shift of the $\mathrm{CV}$ RRC corresponds to an outflow velocity of $-470 \pm 240 \mathrm{~km} \mathrm{~s}^{-1}$ for the recombining $\mathrm{C}$ VI ions. This is in agreement with the average blue-shift of the C vi Ly $\alpha$ line (Fig. 6). However for a complete and spherically symmetric, outflowing shell around the nucleus containing $\mathrm{C}$ VI we would expect to see no net velocity of the RRC. The absence of a red (and zero velocity) component of the RRC can be understood if only a partial shell (opening angle typically 30-60 degrees) is visible. This could be due to some degree of collimation and/or obscuration of the red part by the accretion disk or torus.

\subsection{Low energy emission lines}

In the past there has been a debate whether the EUVE spectrum of NGC 5548 contains emission lines (in particular Ne VII/Ne VIII at $88.1 \AA$ and Si VII at $70.0 \AA$, Kaastra et al. 1995) or that it is a featureless power law (Marshall et al. 1997). Our spectral modeling (see Fig. 16) clearly shows that at long wavelengths the spectrum is not a simple power law or other smooth continuum, but that there are many absorption lines. In fact, our knowledge of the long wavelength lines is currently limited. We expect that many more lines should be present in this energy band than taken into account here, due to other L-shell transitions of ions from $\mathrm{Ne}, \mathrm{Mg}, \mathrm{Si}, \mathrm{S}, \mathrm{Ar}$ and M-shell transitions of iron ions. Our model only contains the strongest of those lines.

We also tested the alternative hypothesis of (thermal) line emission in addition to the modified blackbody and power law continuum. Taking a temperature of $0.06 \mathrm{keV}$, derived from the EUVE data by Kaastra et al. (1995), we find a best-fit emission measure of $(4 \pm 5) \times 10^{70} \mathrm{~m}^{-3}$. This is only $2 \%$ of the emission measure found by Kaastra et al. However for a lower temperature of $0.025 \mathrm{keV}$ we get an emission measure that is consistent with this EUVE value.

We conclude that if the lines seen by EUVE are real, then the emitting component must be time-variable. Our LETGS spectrum has much better spectral resolution then EUVE, but due to the low count rates the signal to noise ratio per resolution element is rather limited. Therefore it is hard to identify individual spectral features beyond $50 \AA$. There are some suggestions for emission features/complexes, e.g. near 79, 82 and $87 \AA$, but we find no obvious identifications. Higher signal to noise data are clearly needed in the long wavelength band in order to resolve this problem.

\section{Discussion}

\subsection{Continuum}

The global shape of the continuum (power law plus soft excess, modeled here by a modified blackbody), is not too different from what has been found in the past, for example using EXOSAT data (Kaastra \& Barr 1989). We will not discuss this here in detail, but only note that the modified blackbody component can be attributed to continuum radiation from the accretion disk. The reason that we expect a modified blackbody instead of a simple blackbody is that Compton scattering in the disk cannot be neglected as compared to free-free absorption. However, at the relatively low temperatures that we find, the absorption opacity is not only caused by free-free absorption, but also free-bound and line opacity are important in the soft X-ray band. This last issue points us to the study of relativistic emission lines from the disk.

\subsection{Relativistic oxygen and nitrogen lines}

We have investigated the possible existence of relativistically broadened emission lines of C, N and O. Similar very strong lines were discovered by Branduardi-Raymont et al. (2001) in MCG -6-30-15 and Mrk 766. We find evidence for such lines (at the $3 \sigma$ confidence level) for $\mathrm{O}$ VIII and N VII. However the line intensity in our case is much weaker, about $20 \%$ of the underlying continuum. In particular, we find equivalent widths of 0.6 and $1.1 \AA$ for both lines, an order of magnitude smaller than the results from the narrow line Seyferts studied by Branduardi-Raymont et al. These authors argued that the lines are produced in the inner, irradiated parts of the accretion disk. We follow this interpretation but do not discuss here in detail the formation process of these lines. We conclude that apparently the emerging line power in NGC 5548 is much smaller than in these other sources. In addition, in NGC 5548 the $\mathrm{C}$ vi line is not detected.

The inclination angle derived from our fit to the $\mathrm{O}$ VIII and N viI Ly $\alpha$ profiles is 46 degrees (Table 1). This is consistent with the results of Yaqoob et al. (2001) for the Fe-K line in their re-analysis of ASCA data including the presence of a narrow emission line as observed with the HETGS of Chandra. In the case of the O VIII and N VII lines we find a rather steep emissivity law $(q=4)$. The inner radius of the disk is not very well constrained, but its upper limit of $2.6 G M / c^{2}$ indicates that the central black hole is more likely of the Kerr than the Schwarzschild type.

Interestingly, Nicastro et al. (2000) in their analysis of BeppoSAX data of NGC 5548 found evidence for a hard to explain, weak excess emission feature with an equivalent width of $2.3 \pm 1.7 \AA$ centered around an observed wavelength of $23 \pm 3 \AA$. Nicastro et a. tried to explain this by an unresolved blend of O VII and O VIII emission lines. To explain the high $E W$ measured, these lines were supposed to be produced in a truly warm gas with a temperature higher than that of photoionization equilibrium. 
Our high-resolution data are incompatible with this explanation. Instead, the centroid and equivalent width of the feature are consistent with our current model for the relativistic nitrogen line.

\subsection{The CVI Lyo line}

We have found no evidence for a relativistic C VI Ly $\alpha$ line. However we found a narrower emission component centered around the rest frame wavelength of this line. There is not much asymmetry in the line profile. Its fitted width $\sigma_{\mathrm{v}}=4500 \pm 1400 \mathrm{~km} \mathrm{~s}^{-1}$ which translates into a $F W H M$ of $10600 \mathrm{~km} \mathrm{~s}^{-1}$. This is consistent with the measured width of the optical broad lines of highly ionized species, such as CIV and He II, which also have widths up to $10000 \mathrm{kms}^{-1}$. Therefore we propose that the broad emission component of $\mathrm{C}$ VI Ly $\alpha$ originates from the same material that also emits the UV/optical broad lines. We note that the average photon flux in the broad C IV line is three orders of magnitude larger than the photon flux we

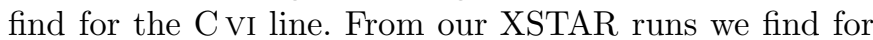
a model with thin irradiated clouds the column density of $\mathrm{C}$ vi to be 1000 times smaller than the C IV column density, if the ionization parameter $\log \xi$ is about -1 . Such an ionization parameter is not unrealistic for the broad line region. Interestingly, for this ionization parameter the $\mathrm{C}$ V column density should be larger than the C vi column density. In principle we might thus also expect a similar emission component for the strongest $\mathrm{C} v$ lines. Inspecting the fit residuals of Figs. 13 and 14 around the $\mathrm{CV}_{1}{ }^{2}-$ $1 \mathrm{~s} 3 \mathrm{p}^{1} \mathrm{P}_{1}$ and $1 \mathrm{~s}^{2}-1 \mathrm{~s} 2{ }^{1} \mathrm{P}_{1}$ lines, at 34.973 and $40.268 \AA$, this is indeed suggested by the data, although for the latter case the significance is restricted by the poor effective area around $40 \AA$.

\subsection{Warm absorber}

Our analysis confirms the dominance of a medium $(\xi \sim$ 100) ionization component B (Kaastra et al. 2000). Component B produces most of the strong absorption lines in the 10-25 $\AA$ band. In addition, we have found evidence for at least two other components at low and high ionization parameter, discussed in more detail below.

\subsubsection{Low ionization component $\mathrm{C}$}

Evidence for the low ionization component $\mathrm{C}$ arises mainly from the blend of weak iron inner shell transitions (unresolved transition array or UTA) in the 16-17 $\AA$ band, as well as from the UV lines in NGC 5548. It is characterized by an ionization parameter 20 times smaller than for component B, and has a significantly smaller column density. How much smaller is hard to say. Table 7 shows a problem with the apparent abundance ratio of iron to the other elements (mainly $\mathrm{C}, \mathrm{N}$ and $\mathrm{O}$ ). Our formal fit in Table 7 needs an overabundance of iron in the low ionization component of 1-2 orders of magnitude as compared to the medium ionization component B. Component B in general does not show too strong deviations from solar abundances.

There are reasons to mistrust this apparent abundance anomaly. In the first place, it is evident from Table 5 that the column densities of $\mathrm{H}, \mathrm{C}, \mathrm{N}$ and $\mathrm{O}$ in the low ionization component are all determined from UV measurements (either FUSE or HST). These observations were not taken simultaneously with the current X-ray data. But even more important than the non-simultaneity is that these abundances are all derived from strongly saturated lines (see the last column of Table 5). In this case the equivalent width of the lines is mainly proportional to the covering factor and the velocity width $\sigma_{\mathrm{v}}$; the dependence upon column density is only logarithmic. This may easily cause strong bias. This was shown by Arav et al. (2002), who demonstrated that the column density of C IV increases by a factor of 4 if covering factors are properly taken into account. However, in our analysis we have accounted for those effects.

On the other hand, the iron abundance of component $\mathrm{C}$ is determined completely by the many weak, unresolved lines of the UTA, all of which have small optical depth. Although the UTA is definitely present, the column densities of the individual ions are sensitive to systematic effects. Small calibration uncertainties may still be present at the few percent level.The relevant wavelength region contains some CsI edges that were taken into account as well as possible. Also, our analysis of the O VII RRC (Sect. 5.7) shows that this feature influences the results for the UTA region. Overall we estimate that these systematic effects will not affect the strength of the UTA by more than a factor of two. They may even increase the UTA's strength.

The weakness of the UTA in the current spectrum makes it hard to determine if component $\mathrm{C}$ is dominated by a single ionization parameter $\xi$, or that it has multiple contributions. In the last case, the slightly different sensitivity to $\xi$ of the UV lines as compared to the iron lines may also cause some differences.

Finally, the modeling of the UV lines only takes into account the resonance absorption of UV photons by the relevant ions. Since the optical depth of the lines is so large, there should also be a significant emission component. Given the complex dynamical structure of the absorber, it is not easy to predict precisely how this will affect the total equivalent width of the absorption line. However if there are significant emission components intermixed with the absorption, the column density of the UV lines may be under-predicted.

Of course component $\mathrm{C}$ could really have a higher iron abundance. Indications for a high iron abundance in another AGN have recently been presented by Boller et al. (2002). They found a sharp drop in the spectrum above $7.1 \mathrm{keV}$ in an XMM-Newton observation of the Narrow-Line Seyfert 1 galaxy 1H 0707-495. In their best model this is interpreted as Fe I K-shell absorption caused by partially covering material with a hydrogen column 
density of a few times $10^{26} \mathrm{~m}^{-2}$ and a strong overabundance of iron by a factor of 35 . This overabundance is similar to what we deduce for component $\mathrm{C}$, however we find a much higher degree of ionization (around Fe XI) and two orders of magnitude smaller column density.

It is evident that spectra with better signal to noise ratio, preferably simultaneously with UV observations are needed to resolve this problem.

\subsubsection{High ionization component $A$}

Evidence for the high ionization component A mainly arises from the strong K-shell resonance absorption lines of S, Si and Mg. Also a major part of the hydrogenic Ne X and O VIII column densities is generated by this component (see Table 5), as well as most of the iron absorption lines from Fe XIX-Fe XXIII. It is puzzling why the apparent iron abundance of this component is so small: iron appears in this component to be under-abundant by a factor of 10 with respect to the other metals.

Table 10. $\sigma_{\text {th }}$ in $\mathrm{km} \mathrm{s}^{-1}$ for the three components for hydrogen, oxygen and iron ions.

\begin{tabular}{lrrr}
\hline \hline Comp. & $\mathrm{A}$ & $\mathrm{B}$ & $\mathrm{C}$ \\
$T(\mathrm{eV})$ & 43 & 8 & 2 \\
\hline $\mathrm{H}$ & 64 & 27 & 14 \\
$\mathrm{O}$ & 16 & 6.9 & 3.4 \\
$\mathrm{Fe}$ & 8.6 & 3.7 & 1.8 \\
\hline
\end{tabular}

A possible explanation is the following. The strong lines of $\mathrm{O}, \mathrm{Ne}, \mathrm{Mg}$ and $\mathrm{Si}$ (dominating the determination of the column density $N$ ) have large optical depths (Table 5). Therefore their equivalent width $E W$ scales like $\sigma_{\mathrm{v}} \ln N$, and thus depends only weakly upon $N$. Most high ionization iron lines have small optical depths, in which case the opposite is true: $E W \sim N$. Therefore the equivalent width does not depend upon $\sigma_{\mathrm{v}}$ but is only proportional to the column density (and thereby the abundance). In our analysis we assumed that all lines have the same value for $\sigma_{\mathrm{v}}$ as the strong UV components, namely $32 \mathrm{~km} \mathrm{~s}^{-1}$. Table 10 shows the thermal contribution $\sigma_{\text {th }}$ to the width for each of the three ionization components. The total width $\sigma_{\mathrm{v}}$ is obtained by combining the thermal width with any turbulent broadening $\sigma_{\text {turb }}$ that is present:

$\sigma_{\mathrm{v}}^{2}=\sigma_{\mathrm{th}}^{2}+\sigma_{\text {turb }}^{2}$

If, for instance, component A has a small turbulent velocity $\sigma_{\text {turb }}$, then $\sigma_{\mathrm{v}}$ for oxygen can be as small as $16 \mathrm{~km} \mathrm{~s}^{-1}$. If that is the case, we need a column density that is two orders of magnitude larger for the strongly saturated ions of $\mathrm{O}, \mathrm{Ne}, \mathrm{Mg}$ and $\mathrm{Si}$ (since $\log N \sim E W / \sigma_{\mathrm{v}}$ ). However for iron we would still obtain the same column density and thus this would worsen the abundance difference. Clearly, the opposite must be true and thus component A must have $\sigma_{\mathrm{v}}$ in the range of $1-2$ times $32 \mathrm{~km} \mathrm{~s}^{-1}$. Kaastra et al. (2000) derived a typical value for $\sigma_{\mathrm{v}}$ of
$140 \pm 30 \mathrm{kms}^{-1}$ for O viI, O viII and Cvi based upon line ratios using lines from the same ion. This was derived under the assumption of a single velocity component. Comparing this width to our current estimate, it follows that we need between $2-5$ velocity components each with $\sigma_{\mathrm{v}}=1-2$ times $32 \mathrm{~km} \mathrm{~s}^{-1}$, to reproduce the total effective width of $140 \mathrm{~km} \mathrm{~s}^{-1}$.

A part of this may be due to the high velocity component $1\left(-1056 \mathrm{~km} \mathrm{~s}^{-1}\right)$. In our analysis we assumed that the ionization component $\mathrm{A}$ is dominated by the velocity components $2-5\left(-669\right.$ to $\left.-163 \mathrm{~km} \mathrm{~s}^{-1}\right)$. For the low wavelength lines we have insufficient spectral resolution to disentangle the velocity components. For C vi we clearly separate component 1 from 2-5 (Fig. 6). Our model predicts that $\mathrm{C}$ VI contains contributions from both components $\mathrm{A}$ and $\mathrm{B}$.

The total column density of component A, derived from the iron lines is approximately $5 \times 10^{25} \mathrm{~m}^{-2}$. This assumes that iron has the same abundance in component $\mathrm{A}$ and $\mathrm{B}$.

\subsubsection{The high velocity component}

The situation around the high velocity component 1 ( $v=$ $-1056 \mathrm{~km} \mathrm{~s}^{-1}$ ) is rather complex and confusing. It was first discovered for N v and C IV lines in Goddard HighResolution Spectrograph (GHRS) observations taken in August 1996 (Crenshaw et al. 1999), and for H I Ly $\alpha$ and N v in March 1998 in STIS spectra (Crenshaw \& Kraemer 1999). The dynamical structure (velocities, widths) did not change over these two years, although the column density decreased by a factor of 4 . Most other velocity components (2-5) did not change that much (except component 3 at $-540 \mathrm{~km} \mathrm{~s}^{-1}$ which decreased by $\sim 40 \%$; this component is the most highly ionized of components 2-5).

FUSE observed NGC 5548 in June 2000 during a low state (Brotherton et al. 2002). It reconfirmed the presence of component 1 in the $\mathrm{OVI}$ and $\mathrm{HI}$ Ly $\beta$ lines. The $\mathrm{HI}$ column reported by Brotherton et al. however is 6 times larger than the column reported by Crenshaw \& Kraemer which was based upon the Ly $\alpha$ observation two years earlier. In Sect. 5.2 we have seen that a reanalysis of Arav et al. (2002) for the $\mathrm{C}$ IV line led to a four times larger column density for component 4 . Since the optical depth of the hydrogen lines is even larger (Table 5), we suppose that the Crenshaw \& Kraemer H I column density was underestimated.

The column densities of O VI and $\mathrm{H}_{\mathrm{I}} \mathrm{Ly} \beta$ for the dynamical component 1 as derived from the FUSE data, are consistent with $\log \xi=2.35 \pm 0.06$ and a hydrogen column density of $1.0 \times 10^{26} \mathrm{~m}^{-2}$, if modeled by XSTAR. This column density is ten times higher than the column derived by Brotherton et al., on the other hand we also have a slightly larger ionization parameter than these authors. For large $\xi$ the OVI and Hi column densities are very sensitive to the details of the photoionization model used. The same model predicts a Si XIV column of $\log N=20.83$, 
smaller than the total observed column of $22.19_{-0.85}^{+0.36}$. But the observed value has (1) a large statistical error due to strong line saturation and (2) it contains contributions from all velocity components. Similar results are obtained for other highly ionized ions such as Mg XII. For C VI the model predicts $\log N=21.30$ (compared to the measured value of $21.32 \pm 0.31$ for the components $2-5$, cf. Table 5 ). Given that component 1 of $\mathrm{C}$ VI contains about a third of the total equivalent line width of the line (Table 3 ), the deviations are not too large. However, the model underpredicts the $\mathrm{N}$ V column by an order of magnitude.

Thus, component 1 has a high ionization parameter. We cannot exclude, however, that it also has a component with a smaller ionization parameter (needed for $\mathrm{NV}$ and C IV), or that it is strongly time variable. It is evident that simultaneous X-ray/UV measurements with high $\mathrm{S} / \mathrm{N}$ are needed to resolve this problem.

\subsubsection{Comparison with previous $\mathrm{X}$-ray observations}

It is not easy to compare the properties of the warm absorber as we derived it here from absorption lines in high resolution LETGS spectra with results obtained by low resolution instruments. Figure 10 illustrates that the wavelength-averaged transmission of the warm absorber in the $12-17 \AA$ band is typically between $0.7-0.8$. However the depth of the continuum absorption edge of O VII $(16.77 \AA)$ is only $9 \%$ and for O VIII (14.23 $\AA$ ) $15 \%$. Effectively, these edges are enhanced by the blends of the UTA from M-shell iron ions and O VIII Ly $\beta$ (for O VII); by blends from L-shell iron ions and neon lines (for O VIII).

This may explain why Reynolds (1997) finds edges of $22 \%$ (O VII) and $15 \%$ (O VIII) in his ASCA data of NGC 5548, somewhat higher than our values. The present results for the dominant ionization component $\mathrm{B}$ are in good agreement with previous studies using ASCA (George et al. 1998) and BeppoSAX (Nicastro et al. 2000). This is demonstrated in Table 11. Ionization parameters used by those authors were converted to our $\xi$ convention using the continuum spectral shape of Sect. 5.5. Both the column densities and the ionization parameters are consistent with the values presented here. It should be noted that the $2-10 \mathrm{keV}$ fluxes during all these observations were rather similar. The HETGS data (taken when the source was two times fainter) also show no evidence for large differences in the warm absorber properties, indicating that component $\mathrm{B}$ is rather stable.

\subsection{Narrow emission lines}

\subsubsection{Intercombination and forbidden lines}

The intercombination and forbidden lines, in the AGN environment formed mainly by recombination, have almost no net outflow velocity. This is in contrast to the strongest $n=2$ to $n=1$ resonance lines from O VII and O viII. These lines have a strong, blue-shifted absorption component with velocities of the order of $-500 \mathrm{~km} \mathrm{~s}^{-1}$,
Table 11. Comparison of the dominant ionization component B.

\begin{tabular}{lrrl}
\hline \hline Instr. & $\begin{array}{r}F_{2-10 \mathrm{keV}} \\
\left(\mathrm{W} \mathrm{m}^{-2}\right)\end{array}$ & $\begin{array}{r}\log N_{\mathrm{H}} \\
\left(\mathrm{m}^{-2}\right)\end{array}$ & $\begin{array}{l}\log \xi \\
\left(10^{-9} \mathrm{~W} \mathrm{~m}\right)\end{array}$ \\
\hline ASCA $^{\mathrm{a}}$ & $4.3 \times 10^{-14}$ & $25.71 \pm 0.05$ & $1.54 \pm 0.03$ \\
ASCA $^{\mathrm{b}}$ & $5.2 \times 10^{-14}$ & $25.51 \pm 0.09$ & $1.90 \pm 0.09$ \\
BeppoSAX $^{\mathrm{c}}$ & $3.5 \times 10^{-14}$ & $25.44 \pm 0.12$ & $2.11 \pm 0.16$ \\
LETGS $^{\mathrm{d}}$ & $4.0 \times 10^{-14}$ & $25.67 \pm 0.17$ & $1.87 \pm 0.17$ \\
\hline
\end{tabular}

\footnotetext{
a Reynolds (1997).

b George et al. (1998).

c Nicastro et al. (2000).

d Present work.
}

and a redshifted emission component with a velocity of $+500 \mathrm{~km} \mathrm{~s}^{-1}$. The blue emission component of these resonance lines cannot originate in the same recombining plasma that produces the non-shifted forbidden and intercombination line. The discussion given by Kaastra et al. (2000) for these last two lines still holds, i.e. from the low value of $i / f$ an upper limit to the density of $7 \times 10^{16} \mathrm{~m}^{-3}$ can be derived. Taking the observed flux of the blue component of the $\mathrm{O}$ VII resonance line as a typical upper limit to the non-shifted flux of this line, the ratio $G=(i+f) / r$ has a lower limit of 3.6. This value is expected for photoionized plasmas and is significantly larger than the ratio for collisional plasmas.

The error bars on the derived fluxes are quite large (the lines are rather weak). For the strongest line (the O VII forbidden line), the intensity did not change much between the LETGS and MEG observations, despite the factor of 2 lower continuum flux in the latter case. This, combined with the low velocity of these lines could indicate that they are formed at a rather large distance from the central ionizing source. Other evidence for the large distance of this component is obtained from the width of the forbidden $\mathrm{O}$ VII line. The line is narrow and consistent with no Doppler broadening. The upper limit to the intrinsic Gaussian line width $\sigma_{\mathrm{v}}$ is $310 \mathrm{~km} \mathrm{~s}^{-1}$ for the LETGS data and $330 \mathrm{~km} \mathrm{~s}^{-1}$ for the MEG data. This would place this component at the same distance as the optical narrow line region.

\subsection{Resonance lines}

The O viI resonance and O viII Ly $\alpha$ line profiles may be considered as a P Cygni type profile of an absorbing wind. The optical depth in the line center of the absorption components of these lines is very large (41 and 85 for O VIII and O viI, respectively, cf. Table 5). This causes the resonantly scattered photons to produce a strong emission component. The fact that we see a red component means that we can look behind the position of the X-ray continuum source in the plane of the sky (we see the receding component of the wind). This implies that the scattering region must be located at a radial distance larger than 
the outer edge of the (presumably optically thick) accretion disk.

Where is the X-ray absorber located? We first note that from a comparison of the X-ray spectra obtained by LETGS and HETGS it is difficult to establish whether the X-ray absorber varies in time, the limitation being mainly statistics. However from the dynamical association with the UV absorbers (in X-rays we see both the high velocity component 1 as well as a blend of absorbers at the same velocities as UV components 2-5) we assume that the X-ray absorber has a similar distance to the central source as the UV absorber. Arav et al. (2002) have argued that the dominant component of the CIV line must be produced somewhere in between the broad and narrow optical line region.

The narrow optical line region in NGC 5548 consists of a low ionization component at about $70 \mathrm{pc}$ distance plus a higher ionization component at a distance of $1 \mathrm{pc}$ ( $\log U=-1.5$, corresponding to $\log \xi=-0.1$ for our assumed spectrum). This last component is still less ionized than our component $\mathrm{C}$ but produces most of the narrow UV emission lines (Kraemer et al. 1998). Thus, the warm absorber must be within a distance of about $1 \mathrm{pc}$.

The high velocity component 1 is strongly variable on the timescale of 1.5 years (Crenshaw \& Kraemer 1999). This puts an upper limit of about 1 light year $\left(10^{16} \mathrm{~m}\right)$ to the size of this component.

Koratkar \& Gaskell (1991) estimated the luminosityweighted radius of the broad C IV emission line to be 31 light days. Similar size were obtained by Dietrich \& Kollatschny (1995). Using a refined reverberation technique, Wanders et al. (1995) found that C IV should be distributed between 5 and 12 light days.

Thus we argue that the absorber is located between 12 light days and 1 light year, i.e. $3 \times 10^{14}-10^{16} \mathrm{~m}$. Since we have shown that the outer radius of the accretion disk must be smaller than the distance to the absorber, the disk must be truncated at a radius less than a light year.

If the wind is launched from the accretion disk, we expect that the gas has a significant rotational velocity component. We see only a redshifted emission component of the resonance lines, and no sign for rotational broadening larger than a few hundred $\mathrm{kms}^{-1}$. This indicates that the gas must somehow have lost much of its initial rotational velocity components.

\section{Summary and conclusions}

We have shown here the power of high resolution X-ray spectroscopy for active galactic nuclei. The warm absorber in NGC 5548 consists of at least three ionization components, labeled here A $(\xi=1000), \mathrm{B}(\xi=100)$ and $\mathrm{C}$ $(\xi=3)$ in decreasing ionization order. The hydrogen column densities of the first two components are $5 \times 10^{25} \mathrm{~m}^{-2}$, and a comparison of the parameters for component $\mathrm{B}$ with published low-resolution data obtained by ASCA and BeppoSAX shows that it is not strongly variable.
Component $\mathrm{C}$ has a total hydrogen column smaller than the other two.

In the UV lines at least six velocity components have been identified in the past. The X-ray lines span the full dynamical range of these components, but due to the limited spectral resolution of the LETGS combined with the present statistics and the lack of simultaneous UV measurements, the precise connection between velocity components $1-6$ and ionization components $\mathrm{A}-\mathrm{C}$ is difficult to quantify. The highest velocity component 1 $\left(-1056 \mathrm{~km} \mathrm{~s}^{-1}\right)$ has at least also a high ionization contribution.

The formal iron abundance of component $\mathrm{A}$ is an order of magnitude too small and that of component $\mathrm{C}$ an order of magnitude to large as compared to the main component B. We argue that a part of this discrepancy may be due to the strong saturation of the absorption lines of the reference ions against which we measure the iron abundance of components $\mathrm{A}$ and $\mathrm{C}$. The relative abundances of the elements as measured in particular for the strong component B are close to solar. Sodium appears to be over-abundant by a factor of two, while N, O and $\mathrm{Si}$ are slightly under-abundant.

The strongest absorption lines (the $1 \mathrm{~s}-2 \mathrm{p}$ lines of hydrogenic and helium-like oxygen) have significant red emission components, with velocities in the range of $+300-+600 \mathrm{~km} \mathrm{~s}^{-1}$. Since these velocities are similar to the average velocity of the blue-ward absorption component, it is evident that we see a major part of the outflowing wind. This means that the outer radius of the accretion disk must be significantly smaller than the typical size of the absorber, for otherwise the disk would occult the red-ward wing of the resonantly scattered oxygen line photons. Combining this with lower and upper bounds for the distance of the absorber, this outer edge must then be smaller than a light year.

The forbidden and intercombination lines of heliumlike oxygen and neon are most likely produces in a region much farther out in the active nucleus, perhaps in or beyond the optical narrow line region. Arguments that support this is the almost zero outflow velocity $\left(-70 \pm 100 \mathrm{~km} \mathrm{~s}^{-1}\right.$ for the O VII forbidden line), the narrow line width (smaller than $310 \mathrm{~km} \mathrm{~s}^{-1}$ ), as well as the fact that the line intensity did not vary significantly in the two months between the LETGS and the HETGS observations, despite the factor of two smaller continuum flux during the latter observation. The same component most likely produces a weak O viI radiative recombination continuum, and possibly also an $\mathrm{RRC}$ from $\mathrm{C}$ V.

We have found evidence for the presence of weak relativistic Ly $\alpha$ emission lines from $\mathrm{O}$ VIII and N vII. These lines are statistically significant but their equivalent width is an order of magnitude smaller than in the narrow line Seyfert galaxies studied by Branduardi-Raymont et al. (2001). The lines are presumably produced in the inner regions of the accretion disk, for which we derive a typical inclination angle of 46 degrees. The inner radius of the disk indicates that the black hole is probably of the 
Kerr type. The nitrogen line was probably also detected (but not identified) in the BeppoSAX data as published by Nicastro et al. (2000).

Further out in the nucleus we find evidence for a broadened ( $F W H M 10000 \mathrm{~km} \mathrm{~s}^{-1}$ ) emission line of C VI. We propose that this line is produced by the broad line region in the same material that also produces the broad component of the UV C IV line. This is suggested by the similar line width and the apparent $\mathrm{C}$ VI to $\mathrm{C}$ IV line ratio.

Thus, we have encountered in NGC 5548 at least four types of emission lines:

- relativistically broadened emission lines from the accretion disk (O VIII and N VII Ly $\alpha$ );

- a highly ionized broad line region line ( $\mathrm{C}$ VI Ly $\alpha$ );

- P Cygni type emission components from the warm absorber (O VIII Ly $\alpha$ and O viI resonance);

- forbidden and intercombination lines from the narrow line region or beyond (O VII, Ne IX).

Acknowledgements. The Laboratory for Space Research Utrecht is supported financially by NWO, the Netherlands Organization for Scientific Research. J.S. Kaastra and D.A. Liedahl acknowledge a NATO Collaborative Research Grant CRG 960027. Work at LLNL was performed under the auspices of the U.S. Department of Energy by the University of California Lawrence Livermore National Laboratory under contract No. W-7405-Eng-48.

\section{References}

Anders, E., \& Grevesse, N. 1989, Geochim. Cosmochim. Acta, 53, 197

Arav, N., de Kool, M., \& Korista, K. T. 2002, ApJ, 566, 699

Behar, E., Kinkhabwala, A., Sako, M., et al. 2001, in Mass outflow in Active Galactic Nuclei, ed. D. M. Crenshaw, S. B. Kraemer, \& I. M. George, ASP Conf. Ser., in press

Behar, E., Sako, M., \& Kahn, S. M. 2001, ApJ, 563, 497

Behar, E., \& Netzer, H. 2002, ApJ, submitted

Boller, T., Fabian, A. C., Sunyaev, R., et al. 2002, MNRAS, 329, L1

Branduardi-Raymont, G., Sako, M., Kahn, S. M., et al. 2001, A\&A, 365, L140

Brinkman, A. C., Gunsing, C. J. T., Kaastra, J. S., et al. 2000, ApJ, 530, L111

Brotherton, M. S., Green, R. F., Kriss, G. A., et al. 2002, ApJ, 565,800
Cowan, R. D. 1981, The theory of atomic structure and spectra (Univ. of California Press, Berkeley)

Crenshaw, D. M., Kraemer, S. B., Boggess, A., et al. 1999, ApJ, 516, 750

Crenshaw, D. M., \& Kraemer, S. B. 1999, ApJ, 521, 572

Dietrich, M., \& Kollatschny, W. 1995, A\&A, 303, 405

George, I. M. 2001, in X-ray Emission from Accretion onto Black Holes, ed. T. Yaqoob, \& J. H. Krolik (http://www.pha.jhu.edu/groups/astro/workshop2001/)

George, I. M., Turner, T. J., Netzer, H., et al. 1998, ApJS, 114, 73

Kaastra, J. S., \& Barr, P. 1989, A\&A, 226, 59

Kaastra, J. S., Roos, N., \& Mewe, R. 1995, A\&A, 300, 25

Kaastra, J. S., Mewe, R., \& Nieuwenhuijzen, H. 1996, in UV and X-ray Spectroscopy of Astrophysical and Laboratory Plasmas, p. 411, ed. K. Yamashita, \& T. Watanabe (Tokyo, Univ. Ac. Press)

Kaastra, J. S., Mewe, R., Liedahl, D. A., Komossa, S., \& Brinkman, A. C. 2000, A\&A, 354, L83

Kallman, T. R., \& Krolik, J. H. 1999, XSTAR photoionization code, ftp://legacy.gsfc.nasa.gov/software/ plasma_codes/xstar/

Kaspi, S., Brandt, W. N., Netzer, H., et al. 2001, ApJ, 554, 216

Koratkar, A. P., \& Gaskell, C. M. 1991, ApJ, 375, 85

Kraemer, S. B., Crenshaw, D. M., Filippenko, A. V., \& Peterson, B. M. 1998, ApJ, 499, 719

Laor, A. 1991, ApJ, 376, 90

Marshall, H. L., Carone, T. E., Peterson, B. M., et al. 1997, ApJ, 479, 222

Mathur, S., Elvis, M., \& Wilkes, B. 1995, ApJ, 452, 230

Mathur, S., Elvis, M., \& Wilkes, B. 1999, ApJ, 519, 605

Nandra, K., Fabian, A. C., George, I. M., et al. 1993, MNRAS, 260,504

Nicastro, F., Piro, L., de Rosa, A., et al. 2000, ApJ, 536, 718

Phillips, K. J. H., Mewe, R., Harra-Murnion, L., et al. 1999, A\&AS, 138, 381

Pradhan, A. K. 2000, ApJ, 545, L165

Reynolds, C. S. 1997, MNRAS, 286, 513

Sako, M., Kahn, S. M., Behar, E., et al. 2001, A\&A, 365, L168

Steenbrugge, K. C., et al. 2002, in preparation

Van der Meer, R., et al. 2002, in preparation

Verner, D. A., \& Yakovlev, D. G. 1995, A\&AS, 109, 125

Verner, D. A., Verner, E. M., \& Ferland, G. J. 1996, At. Data Nucl. Data Tables, 64, 1

Wanders, I., Goad, M. R., Korista, K., et al. 1995, ApJ, 453, L87

Yaqoob, T., George, I. M., Nandra, K., et al. 2001, ApJ, 546, 759 